NASA/TM—2019-220068

\title{
The Effect of Incoming Boundary Layer Characteristics on the Performance of a Distributed Propulsion System
}

Puja Upadhyay

Universities Space Research Association, Cleveland, Ohio

K.B.M.Q. Zaman

Glenn Research Center, Cleveland, Ohio 


\section{NASA STI Program . . . in Profile}

Since its founding, NASA has been dedicated to the advancement of aeronautics and space science. The NASA Scientific and Technical Information (STI) Program plays a key part in helping NASA maintain this important role.

The NASA STI Program operates under the auspices of the Agency Chief Information Officer. It collects, organizes, provides for archiving, and disseminates NASA's STI. The NASA STI Program provides access to the NASA Technical Report Server-Registered (NTRS Reg) and NASA Technical Report ServerPublic (NTRS) thus providing one of the largest collections of aeronautical and space science STI in the world. Results are published in both non-NASA channels and by NASA in the NASA STI Report Series, which includes the following report types:

- TECHNICAL PUBLICATION. Reports of completed research or a major significant phase of research that present the results of NASA programs and include extensive data or theoretical analysis. Includes compilations of significant scientific and technical data and information deemed to be of continuing reference value. NASA counter-part of peer-reviewed formal professional papers, but has less stringent limitations on manuscript length and extent of graphic presentations.

- TECHNICAL MEMORANDUM. Scientific and technical findings that are preliminary or of specialized interest, e.g., "quick-release" reports, working papers, and bibliographies that contain minimal annotation. Does not contain extensive analysis.
- CONTRACTOR REPORT. Scientific and technical findings by NASA-sponsored contractors and grantees.

- CONFERENCE PUBLICATION. Collected papers from scientific and technical conferences, symposia, seminars, or other meetings sponsored or co-sponsored by NASA.

- SPECIAL PUBLICATION. Scientific, technical, or historical information from NASA programs, projects, and missions, often concerned with subjects having substantial public interest.

- TECHNICAL TRANSLATION. Englishlanguage translations of foreign scientific and technical material pertinent to NASA's mission.

For more information about the NASA STI program, see the following:

- Access the NASA STI program home page at http://www.sti.nasa.gov

- E-mail your question to help@sti.nasa.gov

- $\quad$ Fax your question to the NASA STI Information Desk at 757-864-6500

- Telephone the NASA STI Information Desk at 757-864-9658

- Write to:

NASA STI Program

Mail Stop 148

NASA Langley Research Center Hampton, VA 23681-2199 
NASA/TM—2019-220068

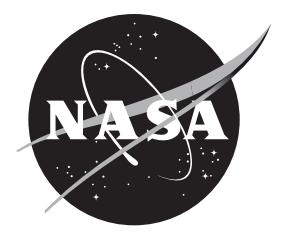

\section{The Effect of Incoming Boundary Layer Characteristics on the Performance of a Distributed Propulsion System}

Puja Upadhyay

Universities Space Research Association, Cleveland, Ohio

K.B.M.Q. Zaman

Glenn Research Center, Cleveland, Ohio

Prepared for the

2019 Science and Technology Forum (SciTech)

sponsored by the American Institute of Aeronautics and Astronautics

San Diego, California, January 7-11, 2019

National Aeronautics and

Space Administration

Glenn Research Center

Cleveland, Ohio 44135 


\section{Acknowledgments}

The authors would like to gratefully acknowledge the support of NASA's Transformational Tools and Technologies (TTT) Project for this research.

This report contains preliminary findings, subject to revision as analysis proceeds.

This work was sponsored by the Transformative Aeronautics Concepts Program.

Trade names and trademarks are used in this report for identification only. Their usage does not constitute an official endorsement, either expressed or implied, by the National Aeronautics and Space Administration.

Level of Review: This material has been technically reviewed by technical management.

Available from

NASA STI Program

Mail Stop 148

NASA Langley Research Center

Hampton, VA 23681-2199
National Technical Information Service 5285 Port Royal Road Springfield, VA 22161 703-605-6000

This report is available in electronic form at http://www.sti.nasa.gov/ and http://ntrs.nasa.gov/ 


\title{
The Effect of Incoming Boundary Layer Characteristics on the Performance of a Distributed Propulsion System
}

\author{
Puja Upadhyay \\ Universities Space Research Association \\ Glenn Research Center \\ Cleveland, Ohio 44135 \\ K.B.M.Q. Zaman \\ National Aeronautics and Space Administration \\ Glenn Research Center \\ Cleveland, Ohio 44135
}

\begin{abstract}
An experimental study is conducted in an effort to advance the understanding of flow physics associated with a boundary layer ingesting, distributed propulsion system. The influence of incoming boundary layer thickness and flow distortions are studied on the flow downstream and the overall performance of the system. The propulsion model, fabricated using additive manufacturing and integrated with electrical fans, is mounted on a flat plate and tested at subsonic speeds. Detailed characterization of the incoming boundary layer and subsequent assessment of the downstream flow field is performed using hotwire anemometry. Modification of the incoming boundary layer is achieved by placing tripping devices, such as rods and vortex generating ramps, near the leading edge of the flat plate. The overall performance of the system for different incoming flow conditions is analyzed by comparing magnitudes of exhaust velocities as well as estimated propulsive power to the corresponding baseline values. For a constant input power to the fans, smaller upstream flow distortions and moderately thickened boundary layers result in marginal changes in the flow field downstream. On the other hand, notable reductions in downstream flow velocities and propulsive power are observed in the case of a significantly thicker and/or distorted incoming boundary layer. It is hoped that this study will serve as a database for this technologically relevant flow field that has not been explored adequately before.
\end{abstract}

\section{Introduction}

The aviation industry is continually striving to develop the next generation of subsonic transport aircraft capable of meeting aggressive targets for fuel efficiency, emissions, noise, and range. While conventional aircraft designs with podded engines have demonstrated greatly improved performance over the years, future aircraft concepts aiming at dramatic leaps in increased efficiency, with improved drag characteristics and lower thrust requirements, heavily rely on propulsion system/airframe integration [1]. One of the key enabling technologies for achieving targeted goals for future subsonic transports is Boundary Layer Ingestion (BLI). The basic principle behind BLI is that a portion of the slow moving boundary layer formed on the aircraft body is ingested, and consequently re-energized, by the propulsion system. In theory, a net gain in overall propulsive efficiency is achieved through lowered drag and corresponding reduction in thrust requirement. This effectively will result in fuel burn and emission benefits [2]. Compared to a pylon mounted engine which takes in freestream air, an embedded propulsion system with BLI reduces the inlet or ram drag which is the force required to decelerate the incoming air before it reaches the fan inlet plane. In addition, a portion of the aircraft wake is ingested by the propulsors. This reduces the overall power requirement, thus providing increased propulsive efficiency.

Theoretical analyses explaining the benefits of boundary layer ingestion on the overall power requirements are discussed by Smith [3] and Drela et al. [4] among others. Plas et al. [2] provided one dimensional analysis comparing BLI and non-BLI propulsors in terms of the total power added to the flow by the engine. For a flow that enters the engine at velocity $u_{i n}$ and exits with velocity $u_{j}$, mechanical power added to the flow is given by Eq. 1 .

$$
P=\frac{\dot{m}}{2}\left(u_{j}^{2}-u_{i n}^{2}\right)=T\left(u_{i n}+u_{j}\right) / 2=T\left(u_{i n}+\frac{\Delta u}{2}\right)
$$

Here, $T=\dot{m}\left(u_{j}-u_{i n}\right)$ is the propulsive force or thrust. Assuming constant mass flow rate, a constant thrust requirement at cruise results in constant $\Delta u=u_{j}-u_{i n}$ term. Therefore, in the case of a BLI engine, it follows from Eq. 1 that a reduced incoming velocity $\left(u_{i n}\right)$ requires lower power added to the flow to produce the same thrust. 
Propulsive efficiency is given by Eq. 2, where $u_{\infty}$ is the free stream speed [5].

$$
\eta=\frac{2 u_{\infty}}{u_{i n}+u_{j}}
$$

For an idealized BLI case, the velocity at the engine exit is assumed to be equal to the free stream speed, $u_{j} \approx$ $u_{\infty}$. Therefore, in principle, a comparatively lower value of the denominator for a BLI engine results in increased propulsion efficiency compared to a non-BLI configuration. Another implication of Eq. 1 is that for a constant thrust, a reduced inlet velocity also results in a lower jet velocity, potentially providing noise benefits. This one-dimensional analysis, although of an idealized case, provides the essence of potential BLI benefits.

One of the key features of aircraft with BLI is the engine placement with propulsors embedded in the airframe at the back of the fuselage. A typical example is the D8 aircraft concept developed by Massachusetts Institute of Technology (MIT) under NASA's N+3 program. This is a collaborative effort among NASA, industry, and academia partners to explore innovative designs and enabling technologies for future subsonic aircraft projected to enter service between 2025-2035 [1, 6, 7]. The D8 'double-bubble' features a wide fuselage for increased lift with engines mounted at the aft end. The design is projected to meet aggressive targets of the $\mathrm{N}+3$ program achieving $70 \%$ reduction in fuel burn and $75 \%$ in emissions compared to the baseline B737-800 aircraft [1]. A recent experimental effort by Uranga et al. [8] compared mechanical flow power required for the BLI version of the D8 aircraft with a corresponding non-BLI design. The former, ingesting about $40 \%$ of the boundary layer, provided a flow power benefit of approximately $8.6 \%$. NASA's STARC-ABL is another recent design that implements BLI on an otherwise traditional airframe by using an aft mounted axisymmetric propulsor $[9,10]$. The electric motor for the propulsor is powered using generators operated by the wing mounted turbo-fan engines. An initial study of this configuration by Welstead and Felder [11] indicated a potential gain in fuel burn saving compared to a conventional propulsion system. The Blended Wing Body (BWB) design studied by Boeing under NASA's Ultra Efficient Engine Technology/ Propulsion Airframe Integration Project is another promising concept with high-lift wings and wide airfoil shaped body with integrated propulsion system [12]. Besides ram drag and viscous drag benefits, system studies of the BWB with BLI have identified other potential sources of performance gains from reduced weight and drag due to elimination of the pylon and a smaller nacelle surface area, better positioning of the thrust reverser, and reduced thrust pitching moment [13]. Another example is a joint effort by NASA Glenn and NASA Langley researchers on a hybrid wing body configuration integrated with a turbo-electric distributed propulsion (TeDP) system. An array of propulsors spanning the entire upper fuselage enable BLI. A comparison with corresponding non-BLI propulsors indicated increased propulsive efficiency with BLI [14]. A numerical study for thrust optimization of the N3-X TeDP configuration with BLI enabling mail-slot propulsion system was also recently carried out by Lee et al.[15]. In general, these system level assessments continue to demonstrate potential benefits of BLI. This motivates research efforts towards proper understanding of this emerging technology and its impact on the next generation of aircraft $[2,16]$.

While the prospect of reductions in fuel burn and emissions for aircraft concepts utilizing BLI is highly promising, it is also realized that the integrated propulsion-airframe architecture is susceptible to numerous limitations that can be detrimental to engine performance. Furthermore, study of such limitations in a highly integrated design is a daunting task requiring system level studies identifying various factors that contribute to overall system performance. Some of the critical issues that are known to arise from ingesting a slow-moving boundary layer include reduced inlet pressure recovery, distorted flow patterns at the engine fan face, and swirl distortions [17]. Complex inlet configurations, such as the one with an S-duct intake, only exacerbate the losses with possible flow separation in the duct [18]. In some cases, reduced fan efficiency or engine performance degradation caused by excessive flow distortion can be severe enough to cause operability and aeromechanical issues, thereby potentially offseting BLI gains [19, 20]. Therefore, a sustainable implementation of BLI technology in future aircraft will greatly depend on two main factors, an optimized inlet design and the ability of the propulsion system to operate under high levels of flow distortions. A significantly improved inlet design by United Technologies Research Center (UTRC) under NASA's Subsonic Fixed Wing Project fulfills multiple objectives for an optimal inlet, maximizing BLI benefits without increasing weight and drag [20]. More recently, NASA, UTRC, and Virginia Polytechnic performed a series of tests with a novel boundary layer ingesting inlet/ distortion-tolerant fan (BLI2DTF) in NASA's $8^{\prime} \times 6^{\prime}$ transonic wind tunnel [21, 22, 23]. Similarly, CFD-based analyses of Liou and Lee [24] for a hybrid wing body and Lee and Liou [10] for the STARC-ABL configuration underscore continued interest in designing optimal geometries to minimize distortion related penalties. In addition to propulsion system optimization, utilization of promising active flow control (AFC) techniques could be crucial for producing acceptable flow uniformity and adequate pressure recovery in the presence of a thick boundary layer. Such benefits of flow control have been experimentally demonstrated in BWB-type configurations [25, 26]. CFD aided studies have also pointed towards the possible benefits of AFC enabled BLI, with some configurations showing potential for up to $10 \%$ reduction in fuel burn [13, 19]. 
The current study takes a fundamental approach towards understanding the flow physics associated with a boundary layer ingesting, distributed propulsion system. In particular, this research effort aims to understand how the incoming flow condition, for example boundary layer thickness and level of distortion, influences the flow behavior downstream of the propulsors. This study is an offshoot of a previous investigation by Zaman and Bridges [27] on 'septa' nozzles. In this previous work, exhaust from an 8:1 converging rectangular nozzle was distributed into six passages using septa inserts in the nozzle. The goal was to study possible adverse effects on noise since jets placed side by side can undergo resonant interactions effectively amplifying noise radiation. The results indicated that noise radiated from the septa nozzle (distributed propulsion) is in fact lower compared to that of the baseline rectangular nozzle. As a follow-up of this previous study [27], small fans were added to the channels and the focus was shifted from noise to aspects of boundary layer ingestion by placing a similar propulsor model on a flat plate inside a wind tunnel. The velocities generated by these fans were low rendering flow field measurements difficult. Subsequently, larger and more powerful fans were incorporated in a larger model leading to the present experiments. Even though the measurements are performed at low Reynolds number conditions, it is hoped that the results would serve as a database for this emerging technology that has not been explored adequately before.

\section{Experimental setup and measurement techniques}

\section{A. Model description}

The experiments are performed in a low-speed, open-loop wind tunnel at NASA's Glenn research center. Detailed information on tunnel operations and capabilities can be found in [28]. The experimental setup is presented in fig. 1 and consists of a five channel, simplified distributed propulsion model mounted on a $16 \mathrm{in} . \times 16 \mathrm{in}$. flat plate fabricated from 1/8th in. thick aluminum stock. The model and plate assembly is fixed to the wind tunnel floor using two vertical support bars and a $10 \mathrm{in.} \times 10 \mathrm{in}$. bottom plate. The leading and trailing edges of the plates and the support bars are chamfered to minimize flow blockage. The overall tunnel test section is $30 \mathrm{in}$. wide and $20 \mathrm{in.} \mathrm{high,} \mathrm{and} \mathrm{therefore}$ negligible wall influences are expected. The origin of the coordinate system, as shown in fig. 1a, is defined at the center of the model exit plane. It should be noted that streamwise distance, $x$, downstream of the model exit plane is defined as positive.

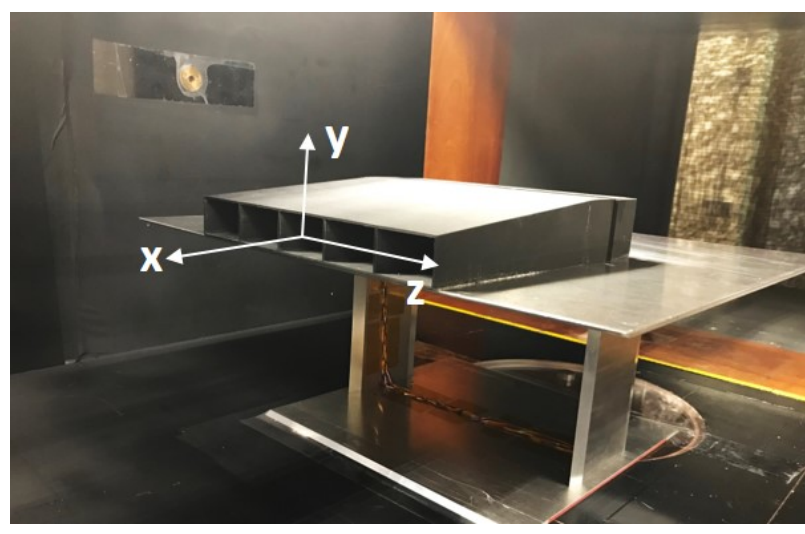

a)

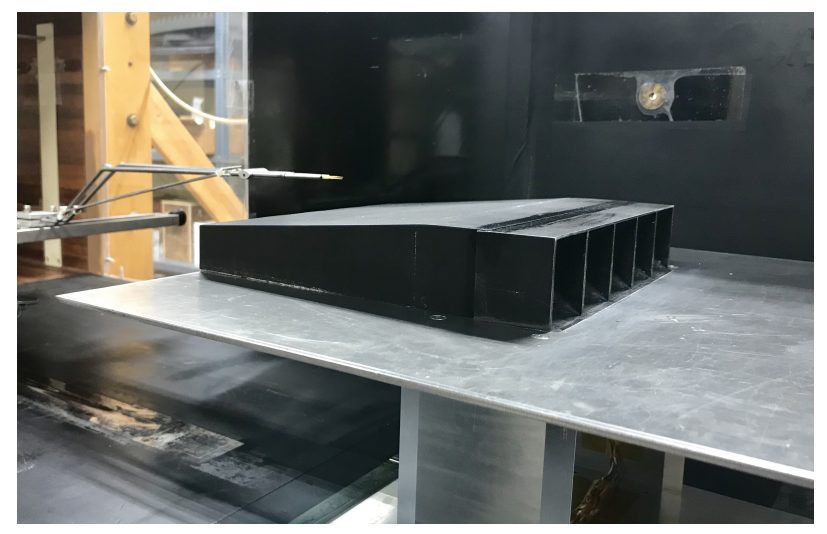

b)

Figure 1: Experimental setup showing (a) the model mounted on the base plate. The origin of the coordinate system is at the center of the model exit. (b) The front view of the model showing the inlets and two X-wires placed side by side.

The model is fabricated using additive manufacturing (3D printing) and integrated with five axial electric fans. The inlet is printed as a separate piece and fitted with the main body using side latches. The mounting plate is designed with a rectangular recess that allows the inlet piece to sit flush with the plate surface. The inlet has five entrances each having a dimension of approximately $1.58 \mathrm{in} . \times 1.58 \mathrm{in} .$. The width $D=1.58 \mathrm{in}$. will be used hereafter for non-dimensionalization of all distances. Each inlet transitions into a circular cross-section guiding the flow to the fan. Downstream of the inlets, each channel houses a fan, where the inlet area of the fan is approximately $1 D \times 1 D$. Flow comes out of the model through rectangular exits with area $A_{e}=1 D \times 0.6 D$. Transition from circular to rectangular cross section is accomplished using contours defined with a third order polynomial. The overall dimension of the model is $8.62 \mathrm{in}$. long in the streamwise direction with a width of $8.2 \mathrm{in}$. The wall thickness is maintained 
between 0.05-0.09 in. depending on locations on the model. Figure 2 shows multiple views of the model with relevant dimensions. Exit channels 1 and 5 are labeled for reference. Although only a simple inlet design is considered in the current study, the interchangeable inlet shown in fig. 2 offers the flexibility to study more complex inlet shapes.

The axial DC fans used in this study are manufactured by Orion fans (Model OD4028-12HBXC01A). Each fan has five blades and seven stators and produces a maximum flow rate of 26.5 cubic feet per minute. Fan rotational speed is a function of supply voltage, where 8 and 10 VDC correspond to 4400 and 5400 revolution per minute (RPM), respectively. A performance curve, flow rate versus static pressure, for the fan model used in this study can be found on the manufacturer's website. A variable power supply unit (Sorensen SRL 20-12) is used to operate the fans at specified voltages. The supply voltage, total current draw, and the rotational speed of the fans during the experiment are monitored using digital multimeters (Keysight model 34461A) and documented for every case.

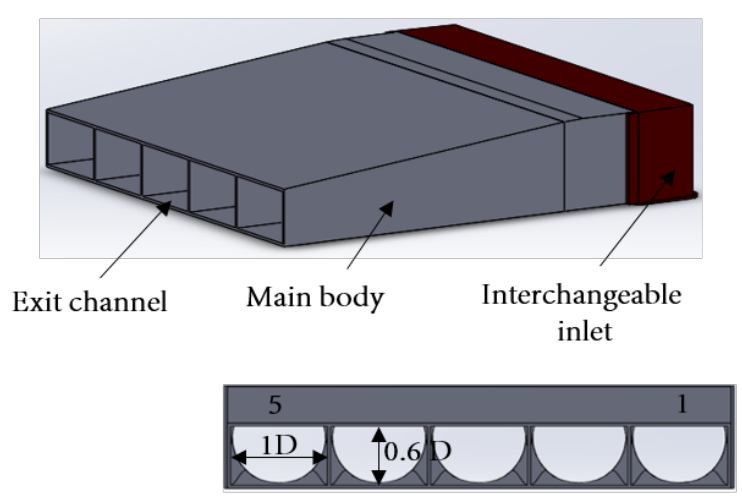

Rear view showing exit channels
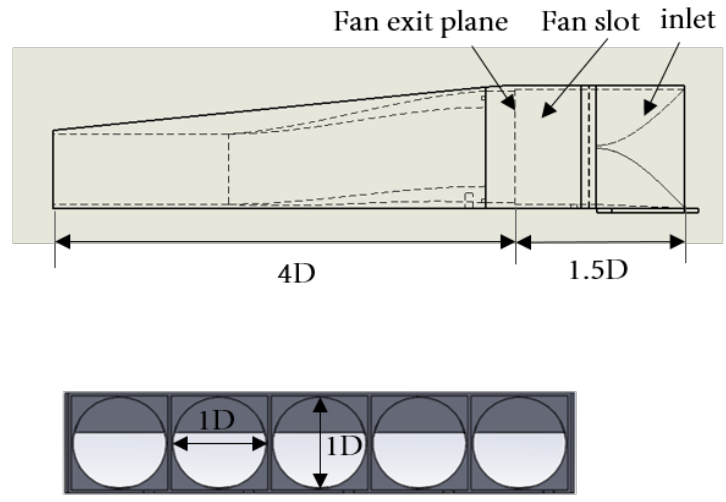

Front view (main body)

Figure 2: Computer aided renderings highlighting different views of the model with relevant dimensions. Exit channels 1 and 5 are labeled for reference.

\section{B. Measurement conditions and techniques}

As discussed in section I, the main objective of this study is to examine the effect of incoming boundary layer characteristics on the performance of the distributed propulsion system. To that end, detailed flow field surveys are performed upstream of the inlet as well as downstream of the model. Figure 3a shows a top view of the model highlighting representative streamwise locations for flow field surveys. A single sensor hotwire probe (TSI 1210-10) is used to perform velocity surveys of the incoming boundary layer at $x=-9.415$ in. $(x \approx-6 D)$. This measurement location corresponds to a distance $0.77 \mathrm{in}$. (0.5D) from the inlet. Each upstream velocity measurement is accompanied by corresponding flow surveys downstream at $x=0.2 D$ and $0.6 D$. Downstream velocity surveys mainly span regions between $y=-0.75 D$ to $0.75 D$ and $z=-3.2 D$ to $1.52 D$, effectively covering channels 2 through 5 . In addition to the single wire, flow downstream is also measured using two X-wire probes (TSI 1241-10), with one in ' $u-w$ ' and the other in ' $u-v$ ' configuration. The probes, separated by a distance of 0.44 in., are traversed through the same grid points in space, and time-averaged values for all three components of velocity $(u, v, w)$ and turbulence intensities $\left(u_{r m s}, v_{r m s}, w_{r m s}\right)$ are obtained. Subsequently, the in-plane velocity vectors ( $w$ and $v$ ) are used to calculate streamwise vorticity $\left(\omega_{x}\right)$. The overheat ratios for the hotwire sensors are maintained between 1.4 and 1.6. Prior to measurements, an in-situ calibration of the probes are performed. The wind tunnel free stream velocity $\left(U_{\infty}\right)$ is calculated using a pitot-static probe.

Flow measurements for this study are performed at two different velocity ratios, $\left(U_{f} / U_{\infty}\right), 1.3$ and 2 . The fan speed $\left(U_{f}\right)$ used in the velocity ratio calculation is the maximum mean velocity achieved by the fans at the model exit plane with no tunnel flow. For a supply voltage $\left(V_{f a n}\right)$ of $8 \mathrm{VDC}, U_{f}$ is approximately $40 \mathrm{ft} / \mathrm{s}$, whereas for $V_{f a n}=10$ VDC, $U_{f} \approx 50 \mathrm{ft} / \mathrm{s}$. For $V_{f a n}=8 \mathrm{VDC}$, a tunnel free stream of $30 \mathrm{ft} / \mathrm{s}$ is chosen, resulting in $U_{f a n} / U_{\infty} \approx 1.3$. This velocity ratio also approximately matches the value in a CFD-based study of a distributed mail-slot propulsion system by Lee et al.[15]. At $U_{\infty}=30 \mathrm{ft} / \mathrm{s}$, in the absence of the model, the Reynolds number at the upstream measurement location $(x=-6 D)$ calculated based on the distance from the plate leading edge is approximately 100000 . With $V_{f a n}$ $=10 \mathrm{VDC}, U_{\infty}$ is set at $25 \mathrm{ft} / \mathrm{s}$ to obtain the other velocity ratio of 2 . 


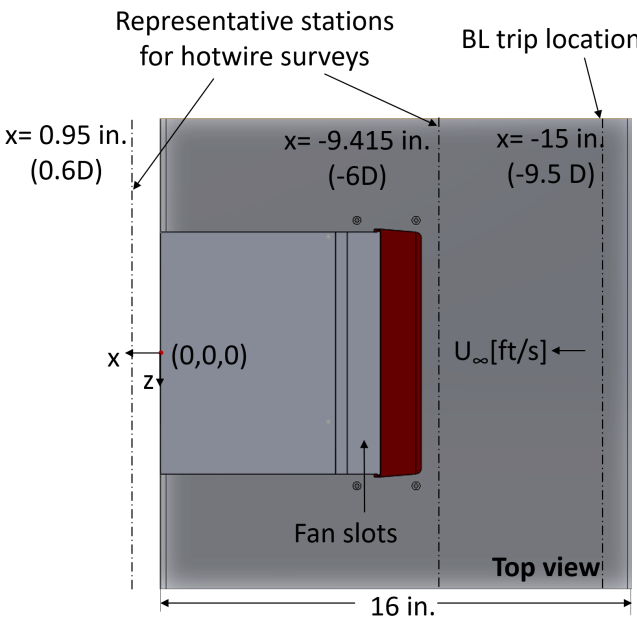

a)

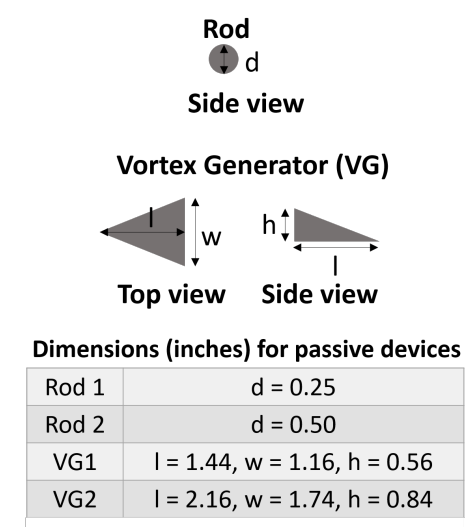

b)

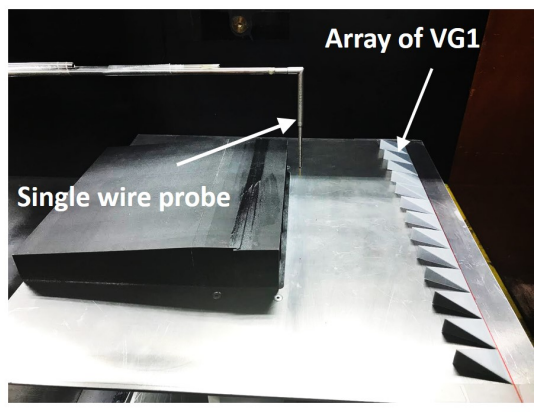

c)

Figure 3: (a) Schematic showing representative locations for hotwire surveys, (b) relevant dimensions of devices used as boundary layer trips, (c) a representative setup showing the position and orientation of an array of vortex generating ramps near the leading edge of the plate.

Lastly, various modifications of the incoming boundary layer are explored in order to understand the influence of upstream conditions on the downstream flow behavior of the system. These modifications are achieved by using boundary layer trips, such as rods and vortex generating ramps of different sizes, placed near the leading edge of the flat plate. Two rods 0.25 in. and 0.5 in. in diameter are used where each rod is placed at $x=-9.5 D$, spanning the entire plate width. Similarly, ramp shaped vortex generators (VG) are placed with their apex facing the model and bases at $x=-9.5 D$. The VG at the center is aligned with the center of the middle inlet channel and the space between two adjacent VGs is kept zero. Relevant dimensions for the boundary layer trips used in this study along with their schematics are presented in fig. 3b. A representative image showing the placement of vortex generators on the flat plate is shown in fig. 3c. As described in the above paragraph, detailed flow surveys using hotwire probes are performed at locations upstream and downstream of the model as shown in fig. 3a. For a given velocity ratio, the power supplied to the fans is kept constant and documented for every case.

\section{Results}

\section{A. Fan flow characterization}

Prior to integration into the BLI model, flow characteristics of an isolated fan are examined at 'wind-off' conditions $\left(U_{\infty}=0\right)$ using a single wire probe. The measurements are performed at supply voltages $V_{\text {fan }}=8$ and 10 VDC and at axial distances $x^{\prime}=0.25 D, 0.6 D$, and $4.6 D$ downstream of the fans, where $x^{\prime}$ is measured from the fan exit plane. Note that in the final configuration, the fan exit plane is approximately $4 D$ upstream of the model exit plane (see fig. 2 and recall that $D=1.58 \mathrm{in}$. is the width of each channel). Figure 4 presents contour maps of time-averaged streamwise velocity $(u, \mathrm{ft} / \mathrm{s})$ for $V_{\text {fan }}=10 \mathrm{VDC}$. The velocity distribution is characterized by regions of high and low velocities along the fan periphery. While the circumferentially periodic deficits are due to the fan struts, the low velocity region in the center can be attributed to flow blockage by the hub. At $x^{\prime}=0.25 D$, the maximum velocity produced by the fan operated at $10 \mathrm{VDC}$ is approximately $60 \mathrm{ft} / \mathrm{s}$. At $V_{f a n}=8 \mathrm{VDC}$, the corresponding maximum velocity drops to about $50 \mathrm{ft} / \mathrm{s}$, not shown for brevity. While streamwise vorticity was not measured for the current isolated fan, previous measurements with a smaller but similar fan revealed no distinct streamwise vortical structures. 


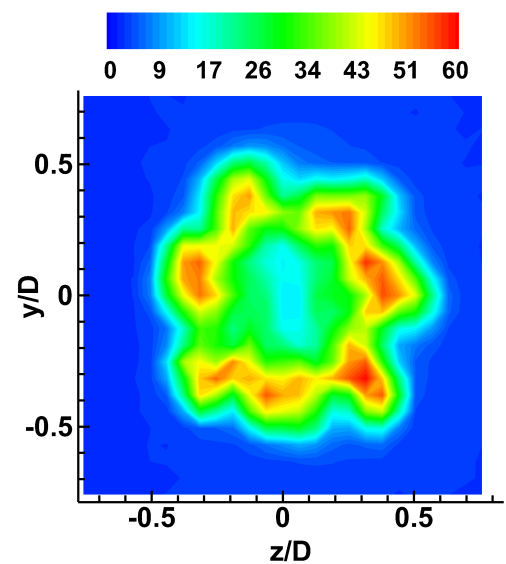

a) $x^{\prime}=\mathbf{0 . 2 5 D}$

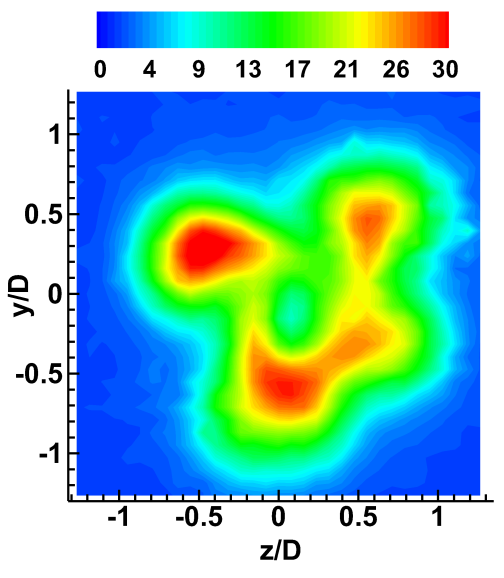

b) $x^{\prime}=\mathbf{2 . 0 D}$

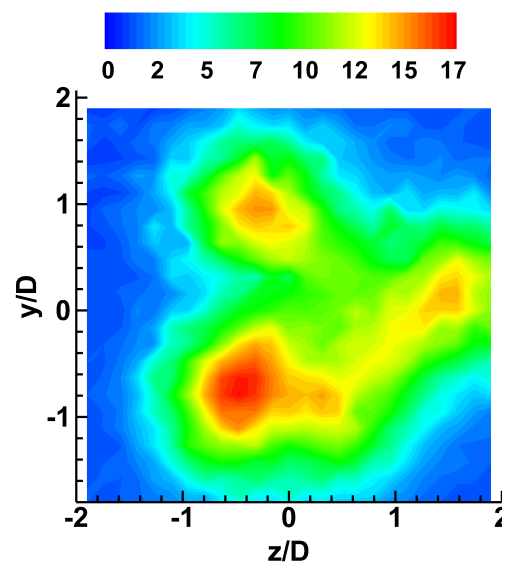

c) $x^{\prime}=4.60 \mathrm{D}$

Figure 4: Streamwise velocity $(u, \mathrm{ft} / \mathrm{s})$ for an isolated fan measured at various streamwise distances $\left(x^{\prime}\right)$ downstream of the fan exit plane. The fans are operated at 10 VDC.

\section{B. Flow field characterization of the BLI model at $U_{\infty}=0$}

The flow behavior downstream of the model was examined with no free stream flow ( $U_{\infty}=0$, 'wind-off' condition). Figure 5 presents contours of mean streamwise velocity measured at axial locations of $x=0.2 D$ and $0.6 D$ for $V_{f a n}=10$ VDC. Relatively coarse grids were used for these measurements in order to capture the full flow field while keeping the survey times reasonably short. In addition, flow surveys with higher spatial resolution were also performed over a limited spanwise range downstream of the middle channel. These contours are included below the primary plots in fig. 5 and provide accurate details of the flow structures. Note that when viewed from downstream, the fan rotation is clockwise.

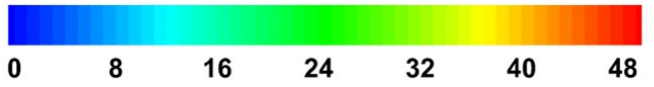

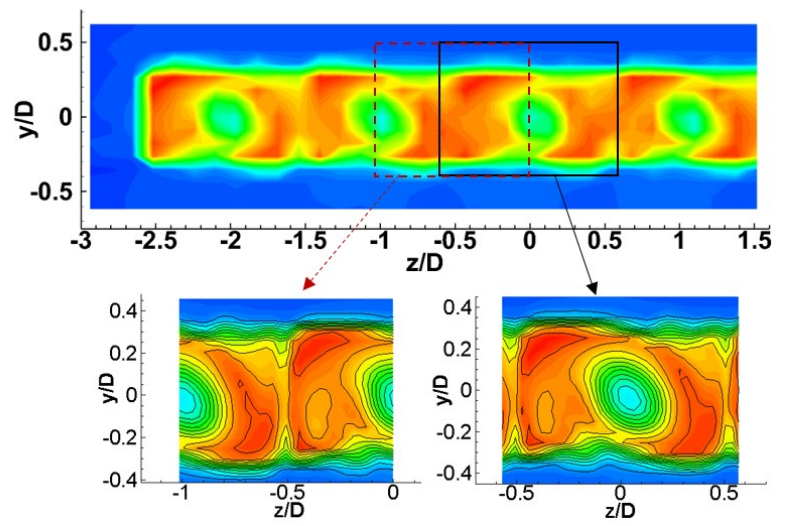

a) $x=0.2 D$

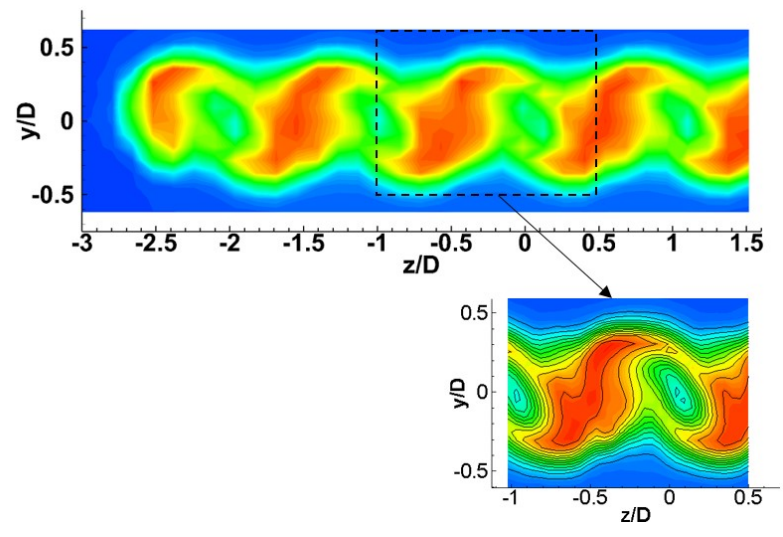

b) $x=0.6 D$

Figure 5: Mean streamwise velocity field $\left(u, \mathrm{ft} / \mathrm{s}\right.$ ) downstream of the model exit plane for $U_{\infty}=0 \mathrm{ft} / \mathrm{s}$ (wind-off condition) and $V_{\text {fan }}=10$ VDC. Measurements with higher spatial resolution around a single exit slot are shown by the insets at the bottom.

At $x=0.2 D$ (see fig. 5a), the mean velocity field downstream of each channel is characterized by a velocity deficit surrounded by high velocity fluid in the peripheral regions. This is observed more clearly in the isolated view for a single channel, as shown by the lower right plot of fig. 5a. As the flow evolves downstream, the velocity field is observed to consist of alternating regions of high and low velocities. The low velocity regions are centered about the channels and, as also observed at $x=0.2 D$, are formed as a result of flow blockage by the hubs. The 


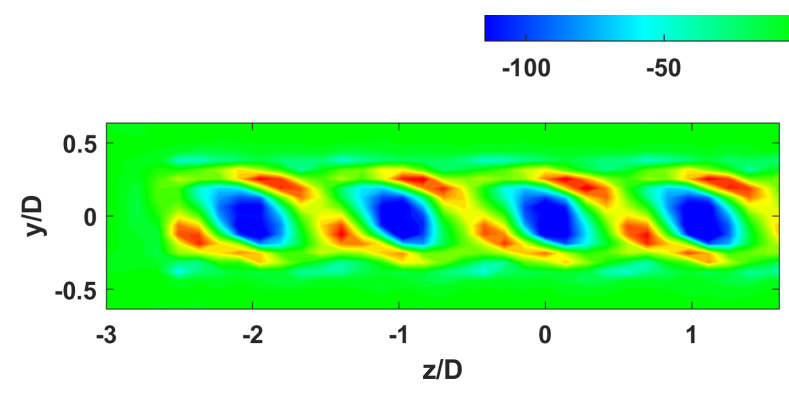

a) $x=0.2 D$
$0 \quad 50 \quad 100$

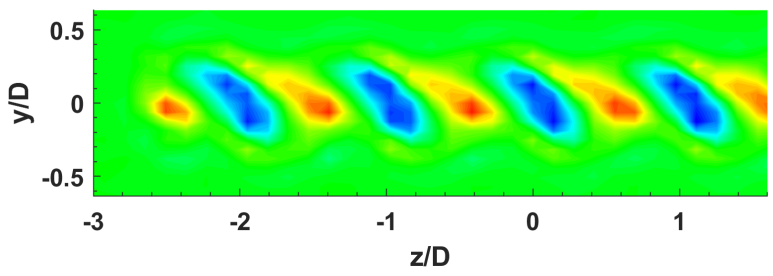

b) $x=0.6 D$

Figure 6: Mean streamwise vorticity $\left(\omega_{x}, \mathrm{~Hz}\right)$ downstream of the model for $U_{\infty}=0$ and $V_{f a n}=10 \mathrm{VDC}$.

high-velocity regions, on the other hand, congregate downstream of the channel partition walls. These high velocity pockets appear to result from the merging or reorganization of high-speed fluid from the adjacent channels. This can also be inferred from the velocity distribution at $x=0.2 D$, particularly as shown by the contour plot in the lower left corner of fig. 5a. The high velocity pocket at the edge of the model (far left) is relatively small since there is no reinforcement from another channel. Moreover, as it will be discussed in the context of fig. 6, the reorganization of the flow field is promoted by the orientation of streamwise vortices that are formed as the flow exits out of the channels. Upon comparison with the measurements for the isolated fan (see fig. 4c), it becomes evident that the flow accelerates through the channels resulting in higher peak velocities compared to that produced by the isolated fan. As noted before, the measurement location $x=0.6 D$ from the model exit corresponds to the location $x^{\prime}=4.6 D$ away from the isolated fan exit plane. Results for $V_{f a n}=8 \mathrm{VDC}$ exhibit very similar behavior, except the magnitudes of velocity are lower at the lower RPM. Fan characteristics including RPM and total power are summarized later.

Figure 6 presents corresponding mean streamwise vorticity distribution at $x=0.2 D$ and $0.6 D$ for $U_{\infty}=0$ and $V_{f a n}=10$ VDC. Closer to the model exit, $x=0.2 D$, formation of negative (clockwise) streamwise structures at the center and positive structures along the edges of the rectangular channels are observed. These vortices are somewhat reminiscent of streamwise vorticity formation in rectangular nozzles due to secondary flow resulting from cross-sectional changes [29]. However, in the current case, the presence of fans imposes additional complexities and the vortices most likely result from some combination of secondary flow in each channel and rotational motion of the fans. As the flow evolves downstream, positive vortex structures from adjacent channels undergo reorganization and merging. The end result is coherent, counter-rotating vortex pairs, as seen in the contour plot at $x=0.6 D$. It can be noted that the positive streamwise structure formed at the edge of the model (far left in fig. 6b) does not have a counter-rotating pair and is also smaller compared to the inner structures. Lastly, a preferential tilt exhibited by the vortices is likely due to the influence of clockwise fan rotation.

\section{Flow characteristics with finite free stream flow}

In this section, flow characteristics in the presence of free stream flow is examined for two different velocity ratios, $U_{f} / U_{\infty}=1.3$ and 2 . Figure 7 presents mean streamwise velocity $\left(u / U_{\infty}\right)$ and turbulence intensity $\left(u_{r m s} / U_{\infty}\right)$ profiles for the incoming flow measured upstream of the inlets at $x=-6 D$ and $z=0$. Vertical dotted lines indicate the surface of the plate and location of the inlet upper lip. In order to avoid accidental breakage of the single wire probe, a small gap measuring approximately 0.03-0.05 in. is maintained between the plate surface and the first measurement location in $y$. For this reason, these measurements can only capture a part of the thin boundary layer formed on the plate surface. Profiles of mean streamwise velocity and turbulence intensity are observed to exhibit similar trends for both velocity ratios. Following a rapid increase in flow speeds away from the plate surface, a region of constant velocity is encountered where velocity magnitudes are lower compared to that of the free stream flow. As will be subsequently demonstrated, flow blockage by the BLI model results in the low velocity region upstream of the inlets. As seen in fig. 7, velocity magnitudes in this region are approximately $80 \%$ and $95 \%$ of the corresponding free stream speeds for $U_{f} / U_{\infty}$ of 1.3 and 2, respectively. Relatively higher velocities for $U_{f} / U_{\infty}=2$ is attributable to increased fan power. At a larger $y / D(>1)$, there is a slight overshoot in the velocity before gradually relaxing to free stream speed. Based on the results, the boundary layer thicknesses are estimated to be $0.19 D$ and $0.17 D$ for $U_{f} / U_{\infty}=1.3$ and 2 , respectively. Profiles for axial turbulence intensity show similar trends with peak values at about $8 \%$ for both velocity ratios. In order to further understand the behavior of the incoming flow, contours of mean streamwise velocity are examined next. 


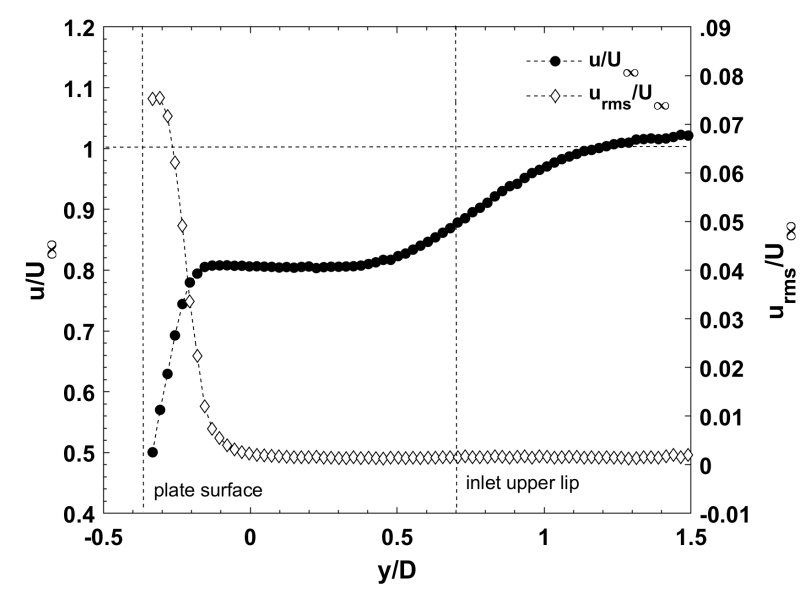

a) $U_{f} / U_{\infty}=1.3$, flat plate

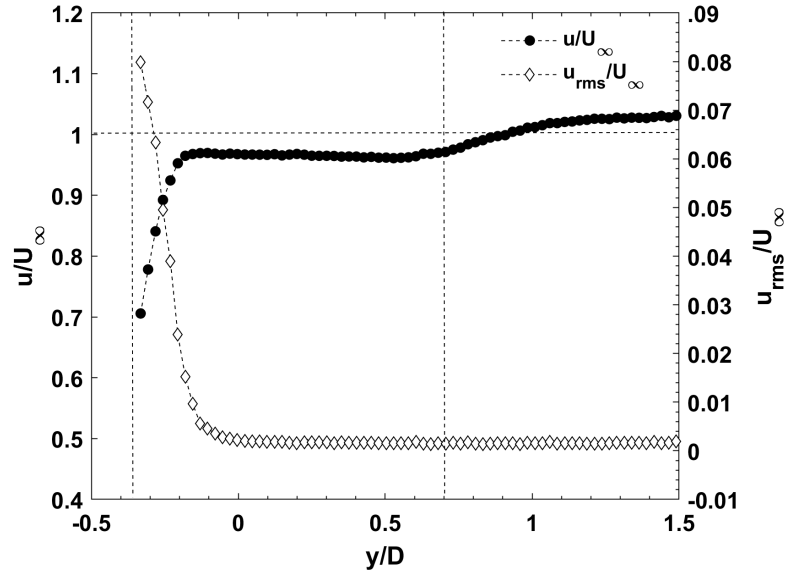

b) $U_{f} / U_{\infty}=2$ flat plate

Figure 7: Incoming boundary layer profiles on the flat plate measured at $x=-6 D$ and $z=0$.

Figure 8 presents contours of mean streamwise velocity upstream $(x=-6 D)$ and downstream $(x=0.6 D)$ of the model. Outlines of the inlets and exit channels are overlaid on the contour plots to identify relative locations of the channels and partition walls. Dotted lines added in the upstream plots represent the plate surface. Flow distribution upstream of the inlet for velocity ratios of 1.3 and 2 can be compared between figs. $8 \mathrm{a}$ and $8 \mathrm{c}$, where formation of thin boundary layer near the plate surface can be observed. In addition, flow blockage by the model results in a 'domeshaped' distribution of velocities; the presence of the model is sensed upstream at these subsonic speeds. As discussed with fig. 7, flow velocities in this region are lower compared to corresponding free stream speeds. An examination of the upstream distribution reveals spanwise variation in velocity magnitudes. Suction by the fans results in pockets of higher velocities upstream of the inlets and lower velocities in the proximity of the partition walls. These spanwise variations are more prominent for the higher velocity ratio, $U_{f} / U_{\infty}=2$, due to increased fan power. For the same reason, the overall normalized velocities in the 'dome' are also higher for this case compared to that for $U_{f} / U_{\infty}=$ 1.3. Downstream of the model, as shown by figs. $8 \mathrm{~b}$ and $8 \mathrm{~d}$, velocity distributions are qualitatively similar to that of the wind-off conditions observed in fig. 5. Moreover, besides the differences in velocity magnitudes, the flow pattern downstream does not exhibit significant dependence on velocity ratio. Figure 9 further presents distribution of turbulence intensity $\left(u_{r m s} / U_{\infty}\right)$ in the streamwise direction at $x=0.6 D$. A similar trend is noted for both velocity ratios with higher $u_{r m s}$ levels localized in regions of high shear.

Figure 10 presents the evolution of mean streamise velocity and vorticity with increasing $x$ downstream of the model. The measurements are performed using two X-wire probes (see section II B) and the velocity results presented here are from the $u w$ probe. As expected, the overall trends captured by the $\mathrm{X}$-wires are similar to that observed with a single wire (see figs. 5 and 8). However, slight smearing or stretching of the flow structures are likely attributable to a finite probe volume and influences from secondary velocity components (e.g., the influence of $v$ component on a $u w$ wire). The $\mathrm{X}$-wire results should be considered qualitative but adequate to show overall distributions of other flow properties not measurable by the single sensor probe. Mean velocity distribution indicate that the flow from adjacent channels interact and evolve rapidly close to the model exit $(x<0.6 D)$. As discussed previously, high velocity flow structures from the adjacent channels merge forming alternating regions of high and low velocities. As the flow evolves further, these high velocity pockets are observed to develop independently with no significant interactions with the neighboring structures. With increasing distances, the structures become diffused with reduced peak velocities. At $x=4 D$, peak velocity is approximately $25 \%$ lower compared to that at $x=0.2 D$.

The development of mean streamwise vorticity (right column in fig. 10) also shows a similar trend. The counterrotating pair of vortices evolve downstream while maintaining their coherent signature. At $x=0.6 D$, the vortex pairs are titled, similar to that observed in fig. 6 for $U_{\infty}=0$. As the flow evolves downstream, the tilt diminishes and disappears by $x=2 D$. Despite reduced amplitudes, the signature of these coherent vortex pairs remain evident even at the farthest measurement station of $x=4 D$ (fig. 10h). While the noise impact of BLI enabled propulsion systems has not yet been explored, the presence of streamwise vortex structures suggests potential acoustic benefit. Introduction of streamwise vorticity has been widely explored before as a means to enhance mixing and reduce noise. The previous study by Zaman and Bridges [27] with septa nozzles also supports this notion. 


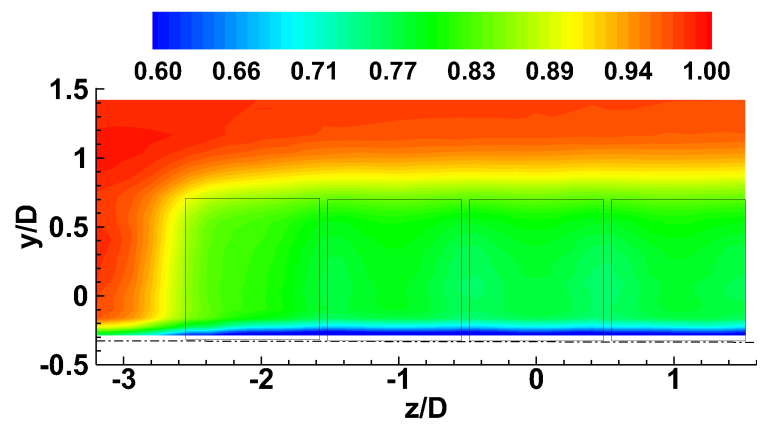

a) $U_{f} / U_{\infty}=\mathbf{1 . 3}, x=-6 D$

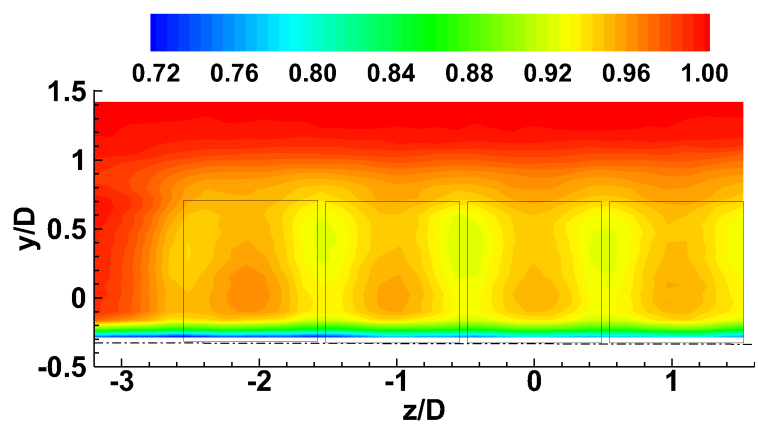

c) $U_{f} / U_{\infty}=\mathbf{2}, x=-6 D$

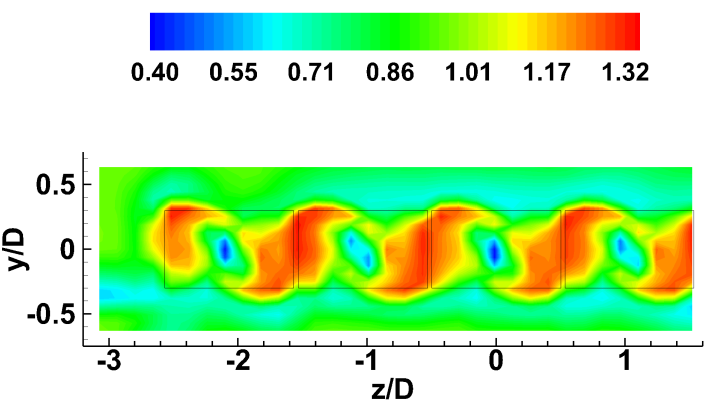

b) $U_{f} / U_{\infty}=\mathbf{1 . 3}, x=0.6 D$
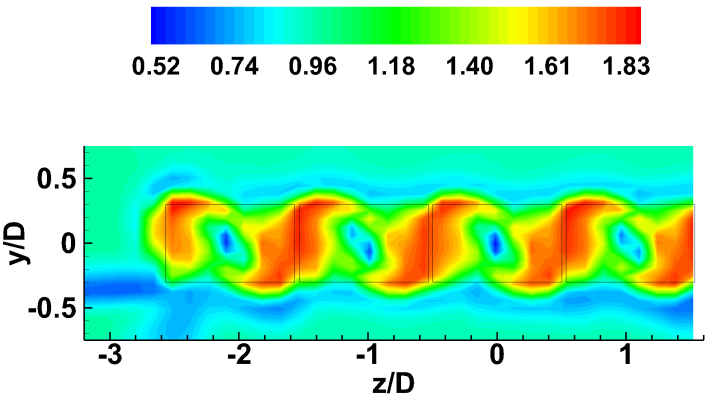

d) $U_{f} / U_{\infty}=\mathbf{2}, x=0.6 \mathrm{D}$

Figure 8: Contours of mean streamwise velocity $\left(u / U_{\infty}\right)$ showing the incoming flow (left) and flow downstream (right) for two different velocity ratios $\left(U_{f} / U_{\infty}\right)$.
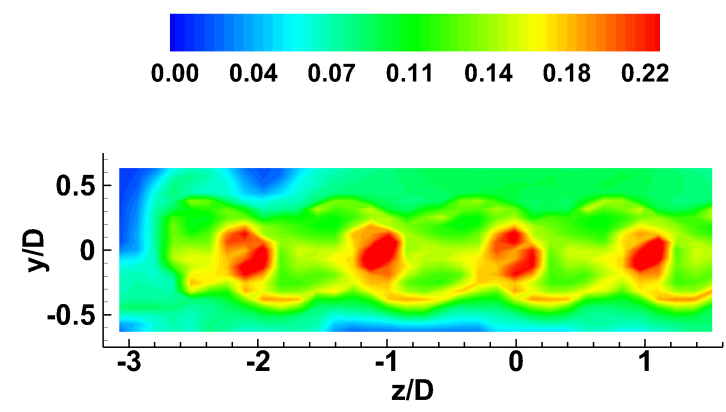

a) $U_{f} / U_{\infty}=\mathbf{1 . 3}$
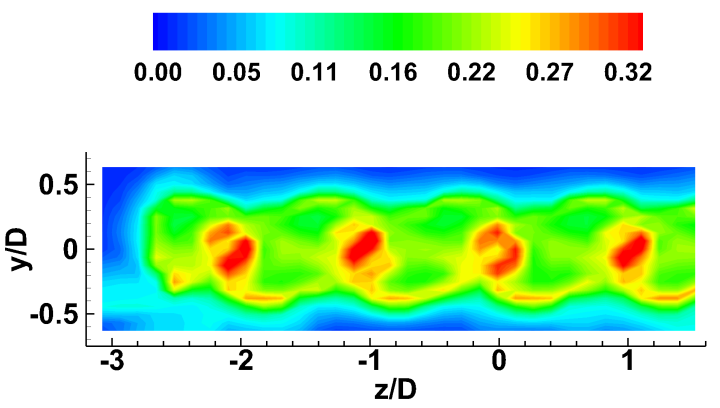

b) $U_{f} / U_{\infty}=\mathbf{2}$

Figure 9: The distribution of turbulence intensity $\left(u_{r m s} / U_{\infty}\right)$ in the streamwise direction downstream of the model at $x=0.6 D$ for two velocity ratios $\left(U_{f} / U_{\infty}\right)$.

For both velocity and vorticity distributions, an interesting trend is exhibited by the structure on the far left side. Due to the absence of a neighboring channel on the left, the high velocity pocket (and the positive streamwise vortex) at the far left is smaller compared to the inner structures. In addition, as the flow evolves downstream, unlike the inner structures that remain centered along the $y=0$ line, it floats away in positive $y$. 


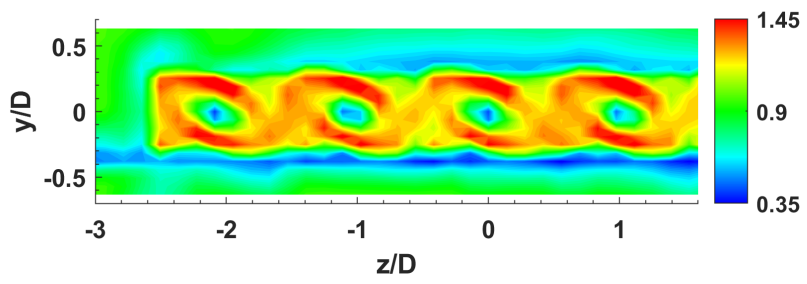

a) $u / U_{\infty}, x=0.2 D$

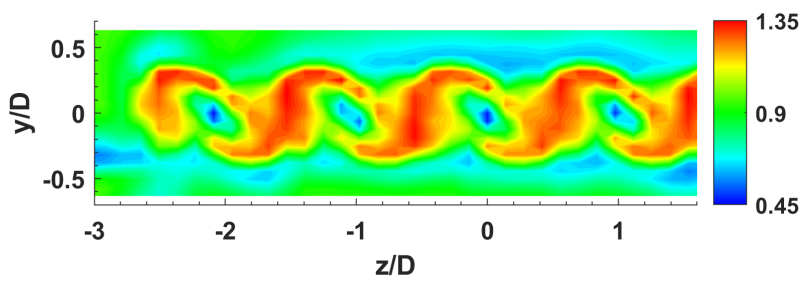

c) $u / U_{\infty}, x=0.6 D$

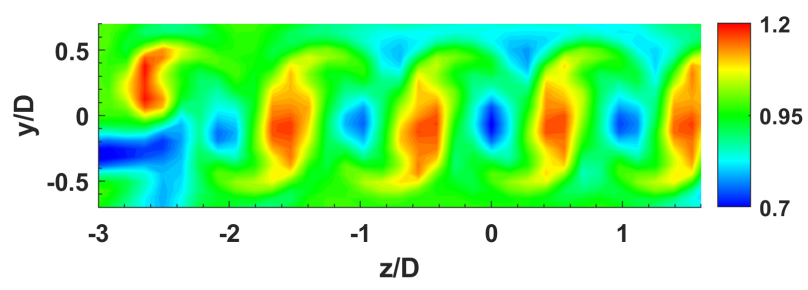

e) $u / U_{\infty}, x=2 D$

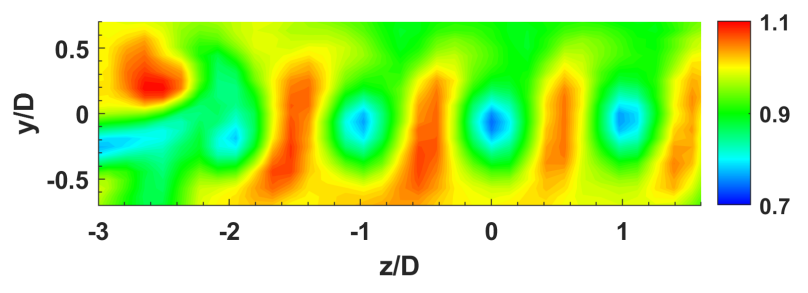

g) $u / U_{\infty}, x=4 D$

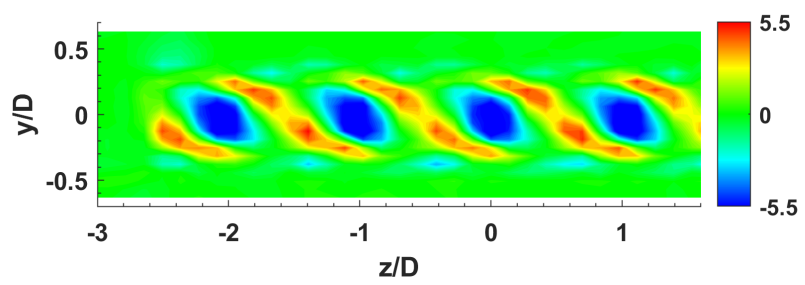

b) $\omega_{x} D / U_{\infty}, x=0.2 D$

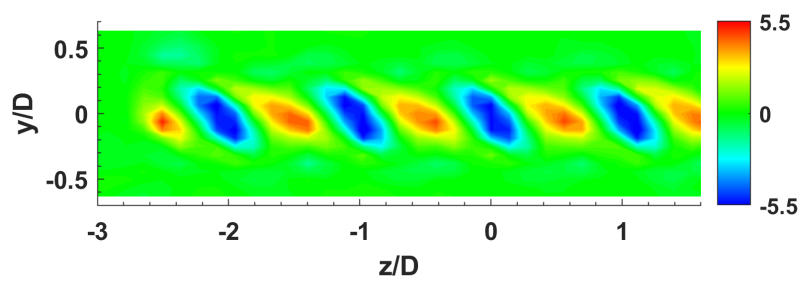

d) $\omega_{x} D / U_{\infty}, x=0.6 D$

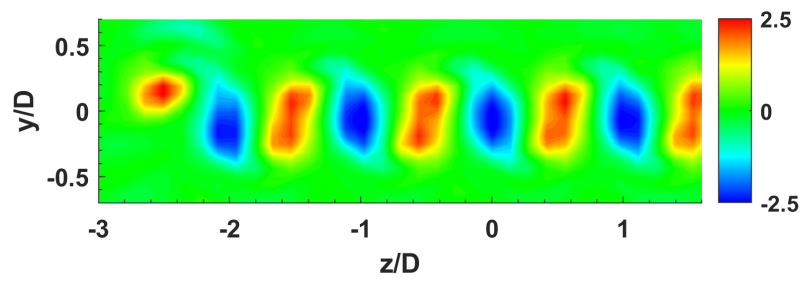

f) $\omega_{x} D / U_{\infty}, x=2 D$

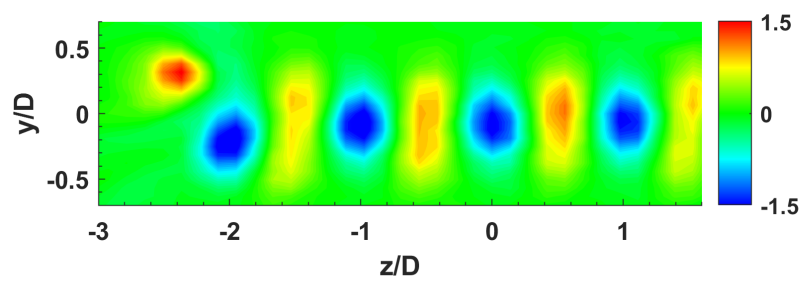

h) $\omega_{x} D / U_{\infty}, x=4 D$

Figure 10: Contours of mean streamwise velocity $\left(u / U_{\infty}\right.$, left column) and vorticity ( $\omega_{x} D / U_{\infty}$, right column) showing the evolution of the flow downstream for $U_{f} / U_{\infty}=1.3$.

\section{Influence of upstream flow modification}

In this section, the effect of incoming boundary layer characteristics on the downstream flow behavior is examined. As discussed in section II B, upstream flow modifications are achieved by placing rods and vortex generating ramps near the leading edge of the flat plate at $x=-9.5 D$. The presence of these passive devices result in an incoming boundary layer that is thicker and/or highly distorted. Figure 11 compares the incoming flow profiles for cases with and without the rods. As in fig. 7, the profiles are measured just upstream of the inlets at $x=-6 D$ and $z=0$. With the rods, a thicker boundary layer is formed resulting in a larger region of low-velocity fluid near the plate surface. As expected, the boundary layer is thicker for the larger $\operatorname{rod}(d=0.5 \mathrm{in}$.). Further away from the plate surface, flow profiles for the tripped cases exhibit increased velocity magnitudes compared to the corresponding baseline values. This local flow acceleration is presumably due to combined effects of flow displacement by the rod and upstream blockage imposed by the model. With the thicker rod, this results in an overshoot with velocity values exceeding the local free stream speed, as shown by the blue profiles. As expected, further away from the model, velocity values for all cases approach free stream speeds.

Figure 12 compares velocity distributions upstream and downstream of the model for various incoming flow conditions. These data, shown similarly as in fig. 8, are for a velocity ratio of 1.3. Even though the baseline (flat plate) case has been discussed earlier in section III C, the results are included for easy comparison. Mean streamwise velocity distribution upstream of the inlets is characterized by a very thin boundary layer near the plate surface accompanied 


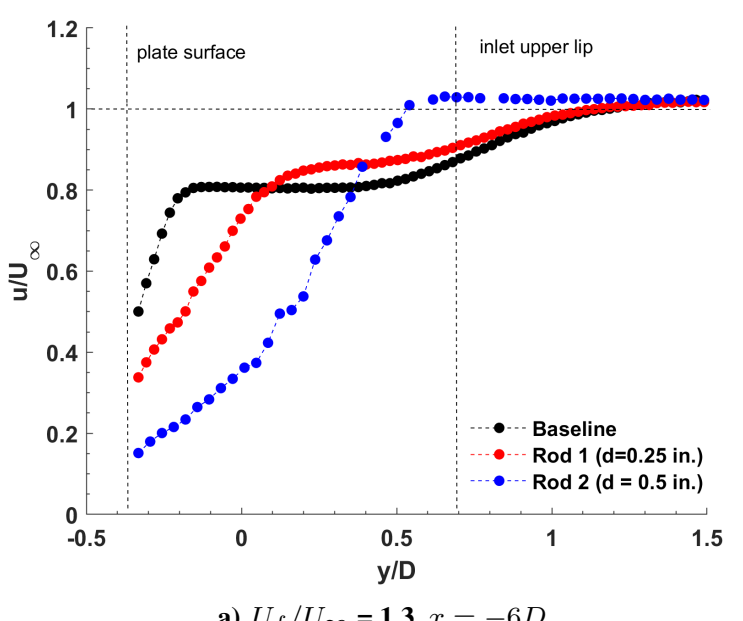

a) $U_{f} / U_{\infty}=\mathbf{1 . 3}, x=-6 D$

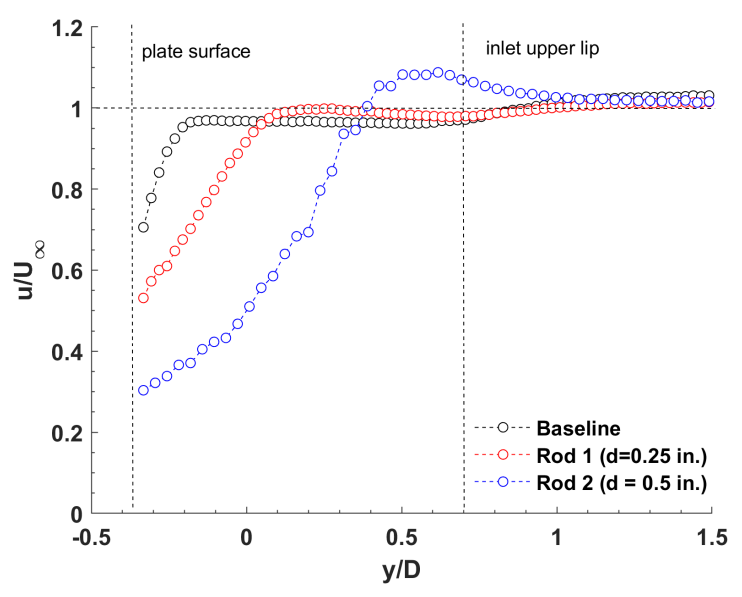

b) $U_{f} / U_{\infty}=\mathbf{2}, x=-6 D$

Figure 11: Comparison of boundary layer profiles upstream of the model inlets for cases with and without the rods.

by a 'dome-shaped' region of relatively constant velocities $\left(u \approx 0.8 U_{\infty}\right)$, as shown in fig. 12a. With the thin rod (d $=0.25 \mathrm{in}$.), the boundary layer thickens with a larger region of low velocity fluid near the plate surface, as shown in fig. 12c. As discussed with fig. 11, further away from the plate, flow velocities higher than the corresponding baseline values are observed due to combined effects of flow displacement by the rod and blockage imposed by the model. With the larger rod $(d=0.5 \mathrm{in}$.) the boundary layer becomes even thicker. In addition, increased flow displacement and local acceleration leads to a much faster transition to free stream, as observed in fig. 12e. The mean velocity contours downstream of the model (right column in fig. 12), show that the overall flow features for the tripped boundary layer cases are similar to that of the baseline flow. As seen in fig. 12d, changes in downstream flow behavior in the presence of the thin rod are limited to minimal reductions in peak velocities. However, with the thicker rod, notable reductions in the velocity amplitudes can be observed (fig. 12f). From these result, it appears that for a fixed input power to the fans, ingestion of a thick boundary layer results in overall reduced exhaust velocities. This suggests increased losses in the fan most likely due to flow non-uniformities at the inlet face and will be further discussed subsequently in the context of flow power.

Upstream flow fields in the presence of two different arrays of ramp shaped vortex generators are shown in figs. $12 \mathrm{~g}$ and 12i. The presence of these ramps not only thickens the boundary layer but also renders it highly distorted. Flow features observed here are in agreement with the results of a previous study conducted using similar vortex generating ramps on a turbulent boundary layer [28]. Counter-rotating pairs of vortices were shown to form downstream of each VG such that an upwash between the two resulted in a 'mushroom' shaped structure. Similar structures are seen in the current results, where larger structures are formed downstream of the larger VGs. The configurations explored here may find significance in examining the response of a BLI system in the presence of a highly distorted boundary layer, for example an integrated propulsion system with an S-duct inlet [18]. The resulting downstream flow fields in the presence of these ramps are shown in figs. $12 \mathrm{~h}$ and $12 \mathrm{j}$. While marginal changes in peak velocity values are realized with smaller VGs, larger vortex generators are found to cause notable reductions in the velocity magnitudes. As with the thicker boundary layer case, these results are suggestive of higher fan losses when there is high level of distortion in the incoming flow.

For the sake of completeness, fig. 13 presents flow comparisons with modified incoming boundary layer for the velocity ratio of 2. Most flow features remain similar to that discussed for the lower velocity ratio, $U_{f} / U_{\infty}=1.3$, in fig. 12. As illustrated in figs. $13 \mathrm{c}$ and $13 \mathrm{e}$, the rods result in thickening of the incoming boundary layer. For the larger rod, increased flow displacement coupled with higher fan power produce flow pockets with velocity magnitudes that are higher than the free stream speed. Upstream flow distribution in the presence of vortex generating ramps (see figs. 13g and 13i) are once again characterized by 'mushroom' shaped structures. Downstream velocity fields exhibit similar trends to that observed for the lower velocity ratio. Overall reductions in exhaust velocities are noted as a result of upstream flow modification with the effects becoming more prominent with increased boundary layer thickness and distortion levels. 


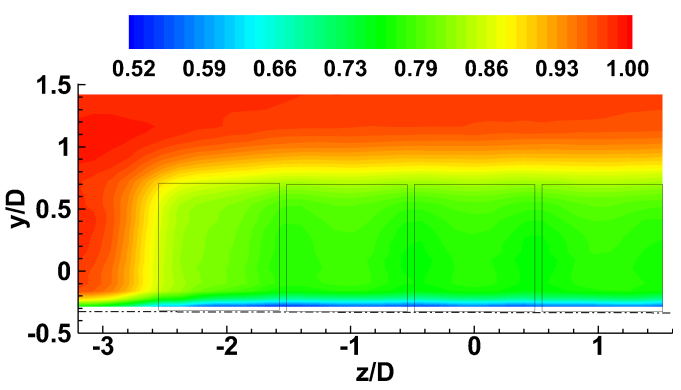

a) flat plate (baseline case), $x=-6 D$

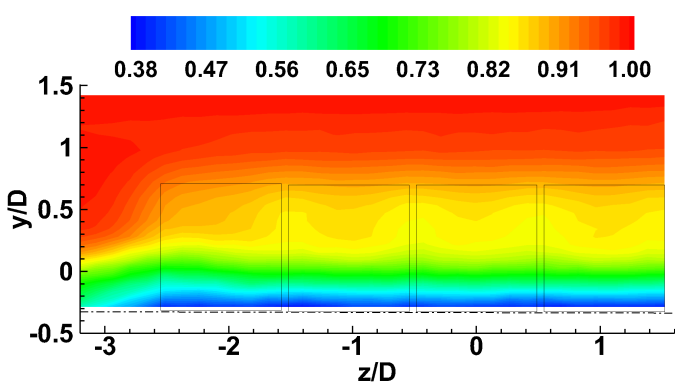

c) $\operatorname{rod} 1(\mathbf{d}=\mathbf{0 . 2 5} \mathrm{in}$.), $x=-6 D$

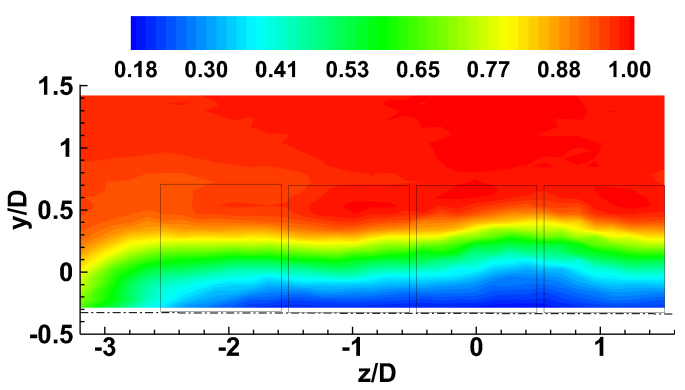

e) $\operatorname{rod} 2(\mathbf{d}=0.5$ in. $), x=-6 D$

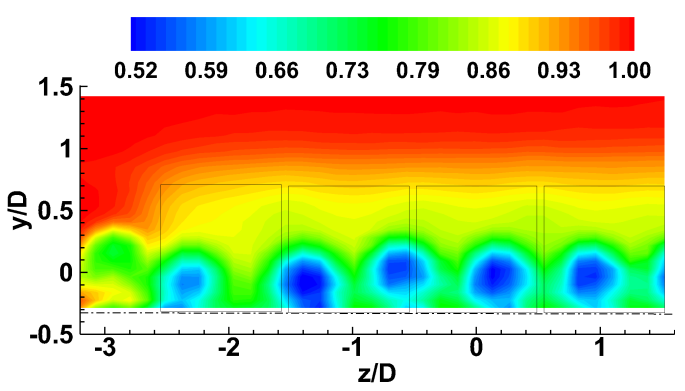

g) VG1, $x=-6 D$

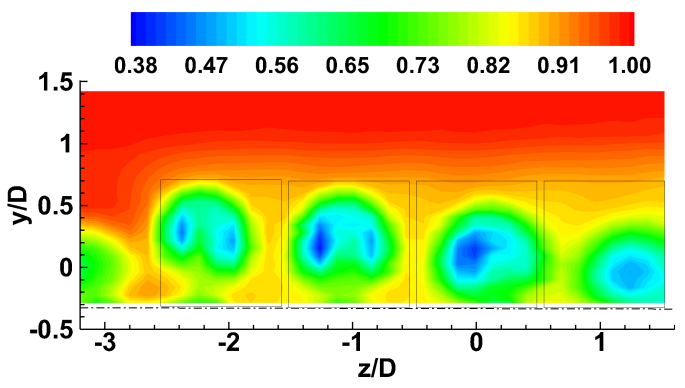

i) VG2, $x=-6 D$

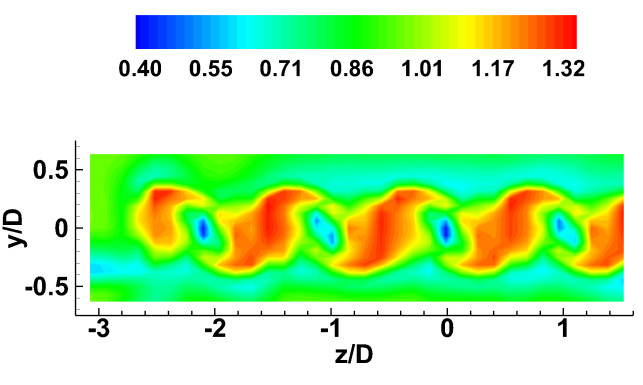

b) flat plate (baseline case), $x=0.6 \mathrm{D}$

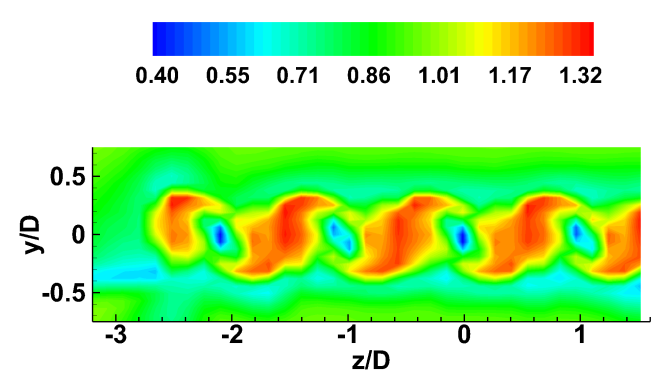

d) $\operatorname{rod} 1(\mathbf{d}=\mathbf{0 . 2 5}$ in. $), x=0.6 \mathrm{D}$

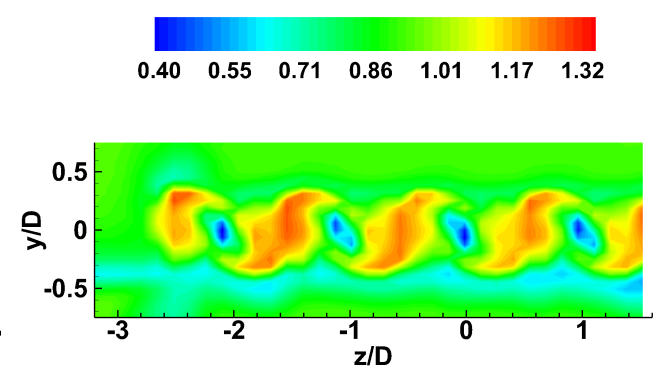

f) $\operatorname{rod} 2(\mathbf{d}=0.5$ in. $), x=0.6 D$

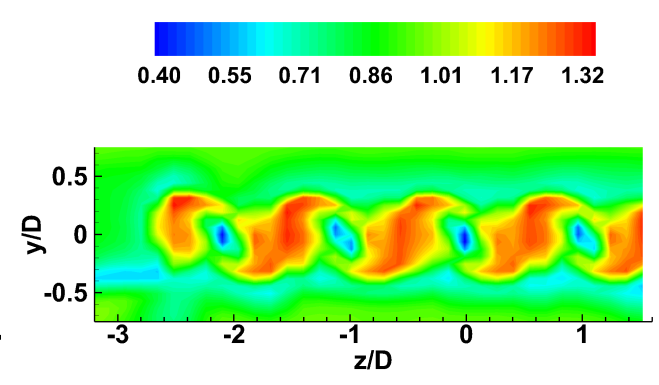

h) VG1, $x=0.6 D$

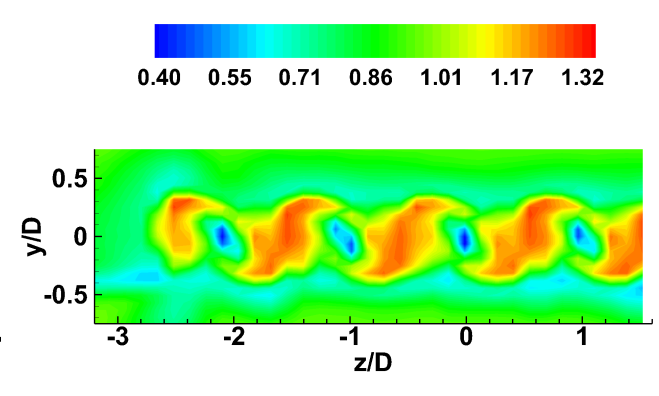

j) VG2, $x=0.6 D$

Figure 12: Contour plots of mean streamwise velocity $\left(u / U_{\infty}\right)$ for $U_{f} / U_{\infty} \approx 1.3$ showing various modifications of the incoming flow (left column) and the corresponding changes in flow behavior downstream of the model (right column). 


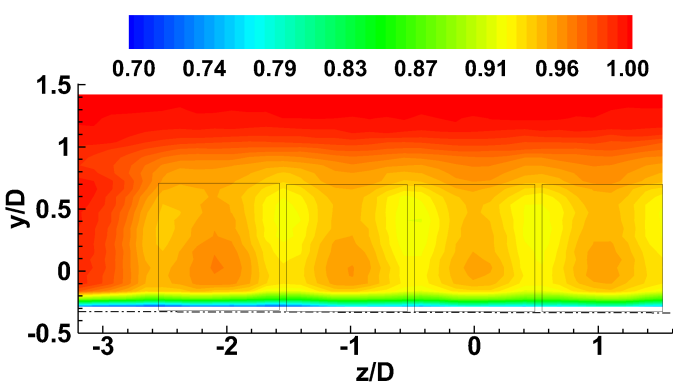

a) flat plate (baseline case), $x=-6 D$

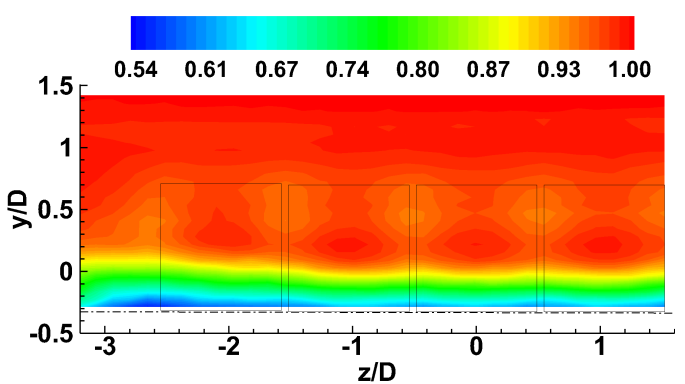

c) $\operatorname{rod} 1(\mathbf{d}=\mathbf{0 . 2 5} \mathrm{in}$.), $x=-6 D$

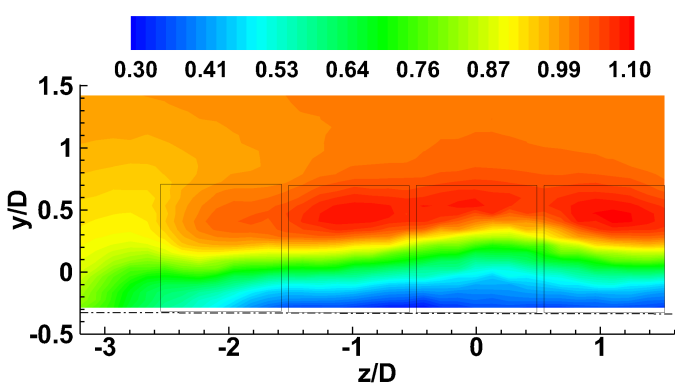

e) $\operatorname{rod} 2(\mathbf{d}=0.5$ in. $), x=-6 D$

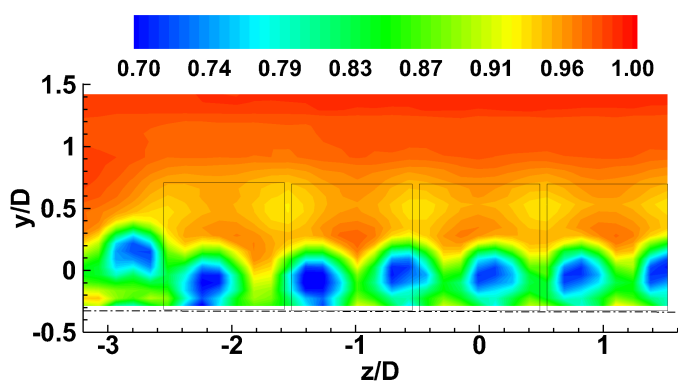

g) VG1, $x=-6 D$

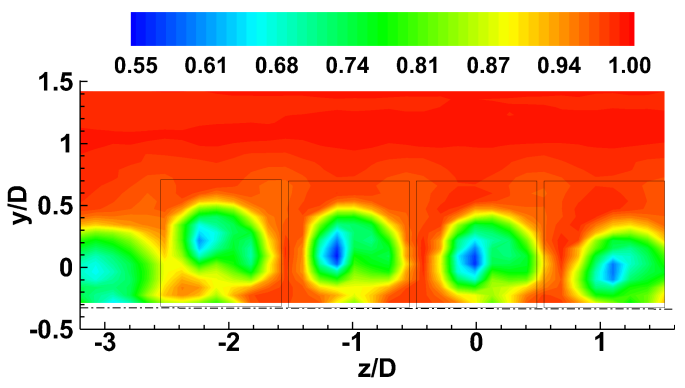

i) VG2, $x=-6 D$

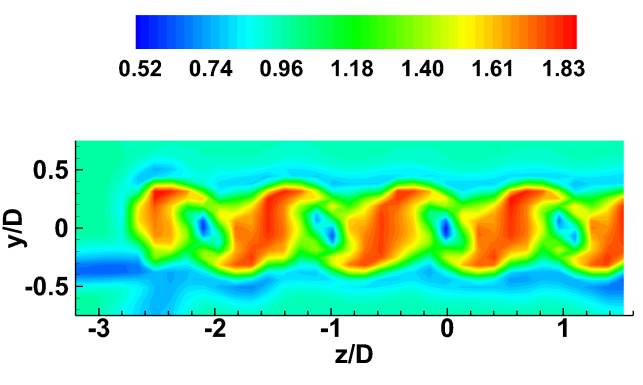

b) flat plate (baseline case), $x=0.6 \mathrm{D}$

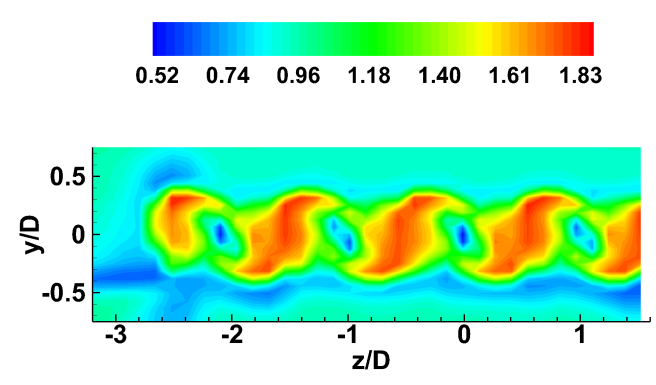

d) $\operatorname{rod} 1(\mathbf{d}=\mathbf{0 . 2 5}$ in. $), x=0.6 \mathrm{D}$

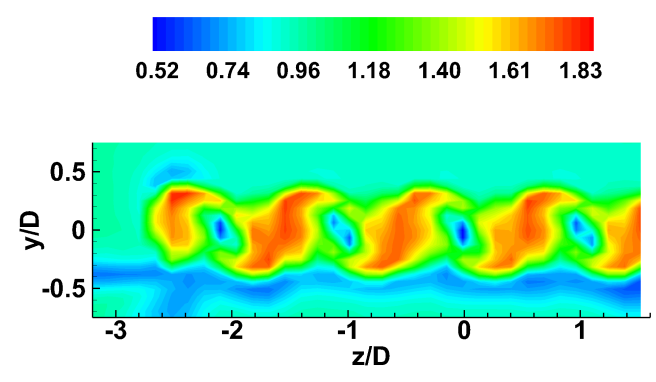

f) $\operatorname{rod} 2(\mathbf{d}=0.5$ in. $), x=0.6 D$

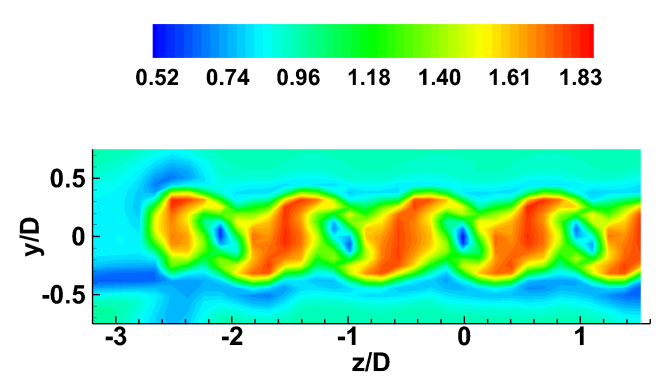

h) VG1, $x=0.6 D$

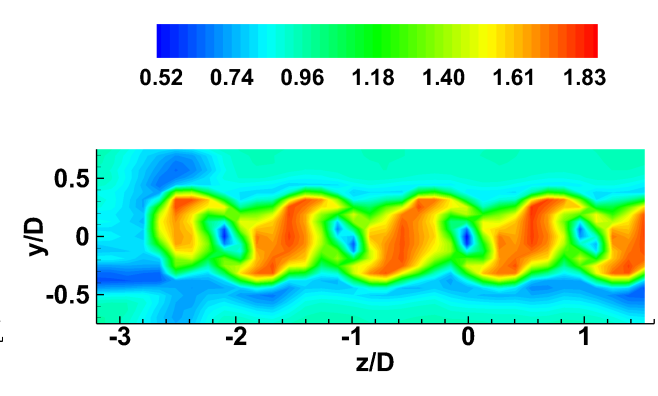

j) VG2, $x=0.6 D$

Figure 13: Contour of mean streamwise velocity $\left(u / U_{\infty}\right)$ for $U_{f} / U \infty \approx 2$ showing various modifications of the incoming flow (left column) and the corresponding changes in flow behavior downstream of the model (right column). 


\section{E. Estimates of power}

Contour plots presented above allow visual inferences regarding the overall behavior of the BLI flow field. A further examination is attempted via the estimation of 'flow power' entering and exiting the BLI model. In this context, flow power is defined as $P_{f}=1 / 2 \rho A u^{3}$, where $\rho$ is the density, $A$ the cross-sectional area over which the power is calculated, and $u$ the mean streamwise velocity. The calculations are performed over channels 2 through 4 , spanning $z$ distances between $\pm 1.52 D$. Velocity field results acquired at upstream $(x=-6 D)$ and two downstream $(x=0.2 D$ and $0.6 D$ ) measurement planes are used. In the upstream measurement plane, the $y$ limits span $-0.29 D$ to $0.71 D$ covering the entire inlet height. In the downstream region, the $\pm y$ limits are defined such that the incoming mass flow rate $\left(\dot{m}=\rho A_{\text {in }} u_{\text {avg,in }}\right)$ equals the outgoing mass flow rate, $\dot{m}=\rho A_{\text {out }} u_{\text {avg,out }}$. With this condition satisfied, flow power is calculated over the same inlet and exit areas. It should be noted that this method only uses the streamwise component of velocity. Possible spanwise and transverse flow exchanges between the primary flow and ambient are not accounted for. In an ideal scenario, mass balance and flow power calculations would need to be performed at the inlet and exit boundaries of the channels which was not possible with the current experimental setup. However, this analysis hopefully provides some useful comparisons between the flow parameters for different incoming boundary conditions. Note that all units here are converted to metric resulting in watts (W) or Joules per $\sec (\mathrm{J} / \mathrm{s})\left[M L^{2} T^{-3}\right]$ as the unit of power. Also, in order to avoid ambiguity, only cases with two dimensional boundary layer, i.e., flat plate and rods, are considered. Table 1 summarizes flow power estimates for velocity ratios of 1.3 and 2 . In addition, total power supplied to the fans ( $P_{f a n}$, watt) for the corresponding cases is also included to facilitate comparison. Furthermore, for all the flow conditions examined, a summary of relevant fan parameters is presented in table 2 .

Table 1: Comparison of flow parameters for different upstream flow conditions

\begin{tabular}{c|c|c|c|c|c|c|c|c}
\hline \hline \multicolumn{4}{c|}{} & \multicolumn{2}{c|}{$x=0.2 D$} & \multicolumn{2}{c|}{$x=0.6 D$} & \\
\hline Case & $U_{f} / U_{\infty}$ & $\begin{array}{c}\dot{m} \\
{[\mathrm{~kg} / \mathrm{s}]}\end{array}$ & $\begin{array}{c}P_{\text {in }} \\
{[\mathrm{J} / \mathrm{s}]}\end{array}$ & $\begin{array}{c}P_{\text {out }, 1} \\
{[\mathrm{~J} / \mathrm{s}]}\end{array}$ & $\begin{array}{c}P_{\text {out }, 1}-P_{\text {in }} \\
{[\mathrm{J} / \mathrm{s}]}\end{array}$ & $\begin{array}{c}P_{\text {out }, 2} \\
{[\mathrm{~J} / \mathrm{s}]}\end{array}$ & $\begin{array}{c}P_{\text {out }, 2}-P_{\text {in }} \\
{[\mathrm{J} / \mathrm{s}]}\end{array}$ & $\begin{array}{c}P_{\text {fan }} \\
{[\mathrm{W}]}\end{array}$ \\
\hline F. plate & 1.3 & 0.0421 & 1.053 & 1.984 & 0.931 & 2.032 & 0.979 & 16.6 \\
Rod 1 & 1.3 & 0.0415 & 1.097 & 1.991 & 0.895 & 2.014 & 0.918 & 16.48 \\
Rod 2 & 1.3 & 0.0352 & 0.969 & 1.572 & 0.603 & 1.559 & 0.590 & 16.39 \\
F. plate & 2 & 0.0421 & 1.046 & 2.809 & 1.763 & 2.742 & 1.696 & 27.10 \\
Rod 1 & 2 & 0.0409 & 0.992 & 2.762 & 1.769 & 2.609 & 1.617 & 27.04 \\
Rod 2 & 2 & 0.0358 & 0.846 & 2.274 & 1.428 & 2.208 & 1.362 & 26.91 \\
\hline
\end{tabular}

Table 2: Relevant fan parameters for various flow conditions examined

\begin{tabular}{c|c|c|c|c}
\hline \hline Case & $U_{f} / U_{\infty}$ & Voltage (VDC) & Fan RPM & $P_{\text {fan }}(\mathrm{W})$ \\
\hline Flat plate & $U_{\infty}=0$ & 8.014 & 4440 & 16.63 \\
Flat plate & $U_{\infty}=0$ & 10.035 & 5340 & 27.3 \\
Flat plate & 1.3 & 8.014 & 4440 & 16.6 \\
Flat plate & 2 & 10.007 & 5340 & 27.1 \\
Rod 1 & 1.3 & 8.012 & 4440 & 16.48 \\
Rod 1 & 2 & 10.009 & 5340 & 27.04 \\
Rod 2 & 1.3 & 8.002 & 4440 & 16.39 \\
Rod 2 & 2 & 10.004 & 5340 & 26.91 \\
VG 1 & 1.3 & 8.011 & 4440 & 16.55 \\
VG 1 & 2 & 10.007 & 5340 & 27.17 \\
VG 2 & 1.3 & 8.006 & 4440 & 16.44 \\
VG 2 & 2 & 10.007 & 5340 & 27.28 \\
\hline
\end{tabular}

Mass flow rates $\left(\dot{m}=m_{\text {in }}=m_{\text {out }, 1}=m_{\text {out }, 2},[\mathrm{~kg} / \mathrm{s}]\right)$ listed for different cases clearly show the velocity deficit imposed by thicker incoming boundary layers. Here, subscripts 1 and 2 denote downstream measurement locations at $x=0.2 D$ and $0.6 D$, respectively. For the thin rod $(d=0.25 \mathrm{in}$.), the overall change in $\dot{m}$ is limited to $1-2 \%$. On the other hand, a thicker boundary layer produced by the larger diameter rod $(d=0.5 \mathrm{in}$.) is observed to reduce the mass flow rates by $15-17 \%$. The 'flow power', calculated in the upstream $(x=-6 D)$ and two downstream planes, $x=0.2 D$ and $0.6 D$, are listed as $P_{\text {in }}, P_{\text {out }, 1}$ and $P_{\text {out }, 2}$, respectively. These show trends that are similar to what is observed for the mass flow rates. A more relevant quantity is the propulsive power (net flow power), calculated as 
the difference between the output and input flow power, $P_{\text {out }}-P_{\text {in }}$. Here, work is done by the fans on the incoming flow resulting in increased flow power downstream. Differences in propulsive power between the baseline and thin rod cases are low, with the rod resulting in a reduction of about $5 \%$ for velocity ratio of 1.3 . With the thicker rod, much larger reductions are noted, where net differences compared to the corresponding baseline cases are approximately $35 \%$ and $19 \%$ for velocity ratios of 1.3 and 2 , respectively.

It is reminded that for all the cases discussed, the total power supplied to the fans is kept constant, as listed under $P_{f a n}$ in table 1. A brief explanation is in order regarding the large difference between the power input to the fans and the net flow power. In an ideal, no loss situation, a net difference in flow power between the upstream and downstream planes would be in the order of the input fan power. However, in the current case, power supplied to the fans are much higher compared to the net flow power $\left(P_{\text {out }}-P_{\text {in }}\right)$ presented in table 1 . Several factors may be responsible for the observed difference. First, power input to the fans $\left(P_{f a n}\right)$ represents total power for five fans whereas flow power calculations only cover channels 2 through 4 . Therefore, only 3/5th of $P_{\text {fan }}$ can be considered for comparison with the estimated propulsive power. Second, fan efficiency which is the aerodynamic ability of the fan to convert mechanical shaft power to air power, comes into play. Losses incurred, such as due to friction and heat, result in a lower net useful power. In addition, flow power calculation should also involve integral of static pressure flux terms at the inlet and exit planes, which are neglected in the current study. This will be attempted in the future to complete the analysis for an extended version of this paper. Lastly, as discussed above, flow exchanges between the primary flow and the ambient between two measurement planes may have resulted in an underestimation of net flow power listed in table 1.

Upon examining the velocity distributions presented in figs. 12 and 13 and flow parameters summarized in table 1, it can be concluded that for the simple system considered here boundary layer ingestion results in a reduced fan efficiency. This is evidenced by overall lowered velocity magnitudes in the downstream flow accompanied by net reductions in propulsive power. These changes, however, are small for moderate upstream flow modifications produced by the thin rod and the smaller vortex generators. For the highly distorted incoming flow with VG2 or the thick boundary layer with the larger rod, reductions in flow velocity downstream and the losses are quite significant. With the thicker rod, the reduction in propulsive power is approximately $35 \%$ (table 1). These observations suggest that the losses in fans are commensurate with the degree of upstream flow modification. Recalling the discussion in section I, the primary benefit of boundary layer ingestion comes from reductions in ram and viscous drag. However, performance penalties are also inevitable due to distorted upstream flow resulting in inlet losses as well as diminished propulsor performance. While the ultimate goal is a net benefit in fuel burn, care must be taken to minimize severe performance penalties that can potentially offset BLI gains and cause operability issues. With the thin rod and smaller vortex generators, small changes in downstream flow despite measurable upstream distortions may show promise in terms of potential reductions in overall drag. The significant loss in propulsive power as a result of larger upstream distortions, for instance with the thicker rod, raises concern about potentially offsetting BLI benefits. However, these are conjectures and no conclusions regarding net gains by BLI can be made without considering a system level study for the entire vehicle. A system level study will take into account benefits from other factors, such as reduction in viscous drag and ram drag, for an embedded propulsor geometry. The merit of the detailed flow field results for the simplified model presented in this paper should be in their usefulness as a database for possible CFD validation of BLI flows.

\section{Conclusion}

A fundamental experimental effort investigating a boundary layer ingesting, distributed propulsion system is conducted. The model, fabricated using additive manufacturing and integrated with five axial electric fans, is mounted on a flat plate and tested at subsonic speeds. The study aims at advancing the understanding of BLI flow physics and exploring the influence of incoming flow characteristics on the overall performance of the system. Detailed surveys of the incoming boundary layer and flow field downstream of the model are carried out using hotwire anemometry. Measurements are performed at two velocity ratios, $U_{f} / U_{\infty}=1.3$ and 2, where $U_{f}$ and $U_{\infty}$ correspond to maximum fan and free stream speeds, respectively. The power supplied to the fans is kept constant for a given velocity ratio. For the baseline cases, a thin boundary layer is measured upstream of the model. Downstream, the flow is characterized by alternating regions of high and low velocity pockets. Strong counter-rotating vortex pairs occur in the flow that remain coherent far downstream from the model. These vortices are formed as a result of some combination of secondary flow in the channels and rotary motion imposed by the fans. Increased flow mixing due to the presence of the streamwise vortices may have potential benefits in the radiated noise levels.

Following the baseline flow studies, the incoming boundary layer is modified by using boundary layer trips, such as rods and vortex generating ramps. The effect of the modified boundary layer on the propulsor is then documented. The incoming boundary layer is made thicker and/or highly distorted by the tripping devices. For the thin rod and smaller 
VGs, despite measurable modifications of the incoming flow, the changes in downstream velocities are marginal. On the other hand, larger upstream distortions imposed by the thicker rod and larger VGs result in notable reductions in the exhaust velocities. A further examination of these results are performed by comparing propulsive power among various cases. Propulsive power is defined as the difference between flow power estimated from mean velocity measured downstream and upstream of the model. While only small changes in propulsive power are noted with the thin rod relative to the baseline case, the thick rod results in approximately $35 \%$ reduction. Even though the current results suggest a reduced propulsor efficiency in the presence of a thicker and/or distorted boundary layer, the net benefits of BLI, for example from reduction in ram and viscous drag, can only be determined with system level assessment of the entire vehicle. It is hoped that the detailed results presented in this study will serve as a database and aid future numerical studies of BLI enabled propulsion systems.

\section{References}

[1] Greitzer, E., Bonnefoy, P., De la Rosa Blanco, E., Dorbian, C., Drela, M., Hall, D., Hansman, R., Hileman, J., Liebeck, R., and Lovegren, J., "N+ 3 Aircraft Concept Designs and Trade Studies, Final Report Volume 1," NASA/CR2010-216794, 2010.

[2] Plas, A., Sargeant, M., Madani, V., Crichton, D., Greitzer, E., Hynes, T., and Hall, C., "Performance of a boundary layer ingesting (BLI) propulsion system," 45th AIAA Aerospace Sciences Meeting and Exhibit, AIAA 2007-450, 2007.

[3] Smith, L. H., "Wake ingestion propulsion benefit," Journal of Propulsion and Power, Vol. 9, No. 1, 1993, pp. $74-82$.

[4] Drela, M., "Power balance in aerodynamic flows," AIAA Journal, Vol. 47, No. 7, 2009, pp. 1761-1771.

[5] Plas, A., Performance of a boundary layer ingesting propulsion system, Master's thesis, Massachusetts Institute of Technology, 2006.

[6] Drela, M., "Development of the D8 transport configuration," 29th AIAA Applied Aerodynamics Conference, AIAA 2011-3970, 2011.

[7] Uranga, A., Drela, M., Greitzer, E. M., Titchener, N. A., Lieu, M. K., Siu, N. M., Huang, A. C., Gatlin, G. M., and Hannon, J. A., "Preliminary Experimental Assessment of the Boundary Layer Ingestion Benefit for the D8 Aircraft," 52nd Aerospace Sciences Meeting, AIAA 2014-0906, 2014.

[8] Uranga, A., Drela, M., Greitzer, E. M., Hall, D. K., Titchener, N. A., Lieu, M. K., Siu, N. M., Casses, C., Huang, A. C., Gatlin, G. M., and Hannon, J. A., "Boundary Layer Ingestion Benefit of the D8 Transport Aircraft," AIAA Journal, 2017, pp. 3693-3708.

[9] Gray, J. S., Mader, C. A., Kenway, G. K., and Martins, J. R., "Approach to Modeling Boundary Layer Ingestion using a Fully Coupled Propulsion-RANS Model," 58th AIAA/ASCE/AHS/ASC Structures, Structural Dynamics, and Materials Conference, AIAA 2017-1753, 2017.

[10] Lee, B. J. and Liou, M.-F., "Aerodynamic Design and Optimization of Fan Stage for Boundary Layer Ingestion Propulsion System," The 10th International Conference on Computational Fluid Dynamics (ICCFD10), Barcelona, ICCFD10-091, 2018.

[11] Welstead, J. R. and Felder, J. L., "Conceptual design of a single-aisle turboelectric commercial transport with fuselage boundary layer ingestion,” 54th AIAA Aerospace Sciences Meeting, AIAA 2016-1027, 2016.

[12] Liebeck, R. H., "Design of the blended wing body subsonic transport,” Journal of aircraft, Vol. 41, No. 1, 2004 , pp. 10-25.

[13] Daggett, D. L., Kawai, R., and Friedman, D., "Blended wing body systems studies: boundary layer ingestion inlets with active flow control," NASA/CR-2003-212670, 2003.

[14] Felder, J. L., Kim, H. D., Brown, G. V., and Chu, J., "An examination of the effect of boundary layer ingestion on turboelectric distributed propulsion systems," 49th AIAA aerospace sciences meeting including the new horizons forum and aerospace exposition, AIAA 2011-300, 2011.

[15] Lee, B. J., Liou, M.-F., and Kim, H., "Design of Distributed Mail-slot Propulsion System on a Hybrid Wingbody Aircraft," 2018 Applied Aerodynamics Conference, AIAA AVIATION Forum, AIAA 2018-3954, 2018.

[16] Hardin, L. W., Tillman, G., Sharma, O. P., Berton, J., and Arend, D. J., "Aircraft System Study of Boundary Layer Ingesting Propulsion," 48th AIAA/ASME/SAE/ASEE Joint Propulsion Conference \& Exhibit, AIAA 2012-3993, 2012.

[17] Seddon, J. and Goldsmith, E., Intake Aerodynamics, AIAA education series, Blackwell Science, 1999.

[18] Berrier, B. L., Carter, M. B., and Allan, B. G., "High Reynolds number investigation of a flush-mounted, S-duct inlet with large amounts of boundary layer ingestion,” NASA/TP-2005-213766, 2005. 
[19] Kawai, R. T., Friedman, D. M., and Serrano, L., "Blended wing body (BWB) boundary layer ingestion (BLI) inlet configuration and system studies," NASA/CR-2006-214534, 2006.

[20] Florea, R. V., Matalanis, C., Hardin, L. W., Stucky, M., and Shabbir, A., "Parametric analysis and design for embedded engine inlets," Journal of Propulsion and Power, Vol. 31, No. 3, 2015, pp. 843-850.

[21] Arend, D. J., Tillman, G., and O’Brien, W., "Generation after next propulsor research: Robust design for embedded engine systems," 48th AIAA/ASME/SAE/ASEE Joint Propulsion Conference \& Exhibit, AIAA 2012-4041, 2012.

[22] Arend, D. J., "Experimental Evaluation of BLI Propulsion: The Boundary Layer Ingesting Inlet/Distortion-Tolerant Fan Solution," AIAA Propulsion and Energy Forum, Techincal Presentation, 2018.

[23] Hirt, S. M., Wolter, J. D., Arend, D. J., Hearn, T. A., Hardin, L. W., and Gazzaniga, J. A., "Performance Calculations for a Boundary-Layer-Ingesting Fan Stage from Sparse Measurements," 2018 AIAA Aerospace Sciences Meeting, AIAA 2018$1889,2018$.

[24] Liou, M.-S. and Lee, B. J., "Minimizing inlet distortion for hybrid wing body aircraft," Journal of Turbomachinery, Vol. 134, No. 3, 2012, pp. 031020.

[25] Gorton, S. A., Owens, L. R., Jenkins, L. N., Allan, B. G., and Schuster, E. P., "Active flow control on a boundary-layeringesting inlet," 42nd AIAA Aerospace Sciences Meeting and Exhibit, AIAA 2004-1203, 2004.

[26] Owens, L. R., Allan, B. G., and Gorton, S. A., "Boundary-layer-ingesting inlet flow control," Journal of Aircraft, Vol. 45, No. 4, 2008, pp. 1431-1440.

[27] Zaman, K. B. M. Q. and Bridges, J. E., "Flow and noise from septa nozzles," International Journal of Aeroacoustics, Vol. 17, No. 1-2, 2018, pp. 36-51.

[28] Zaman, K. B. M. Q., Hirt, S. M., and Bencic, T. J., "Boundary Layer Flow Control by an Array of Ramp-Shaped Vortex Generators," NASA/TM-2012-217437, 2012.

[29] Zaman, K. B. M. Q., "Axis switching and spreading of an asymmetric jet: the role of coherent structure dynamics," Journal of Fluid Mechanics, Vol. 316, 1996, pp. 1-27. 


